\title{
Large Elastoplastic Deformation of a Sample under Compression and Torsion in a Rotational Diamond Anvil Cell under Megabar Pressures
}

\author{
Biao Feng, ${ }^{1}$ and Valery I. Levitas, ${ }^{2, *}$ \\ 1) Department of Aerospace Engineering, Iowa State University, Ames, Iowa 50011, USA \\ 2) Departments of Aerospace Engineering, Mechanical Engineering, and Material Science \\ and Engineering, Iowa State University, Ames, Iowa 50011, USA
}

\begin{abstract}
Material behavior under high pressure and large plastic shear is usually studied in a rotational diamond anvil cell (rotational DAC, or RDAC). The first modeling results for threedimensional plastic flow and evolution of the stress-strain state for a rhenium sample compressed and twisted in RDAC under megabar pressures are presented. Large elastic and plastic deformation theory is utilized. In addition to quantitative information about evolution of the stress-strain fields, the following unexpected results are obtained. During initial compression followed by torsion under a fixed axial force and for beveled anvils, the sample thickness reduces by a factor of ninety. Pressure, as well as the pressure gradient, grow drastically at the central part, and this new phenomenon is called the pressure self-focusing effect. This effect allows a much higher maximum pressure to be reached in a RDAC than in a DAC under the same force. The effects of diamond and sample shape, yield strength and its pressure dependence, the friction coefficient, and applied force on the pressure distribution are analyzed. The obtained results open new ways to increase maximum achievable pressure and develop new intuition in mechanical responses at megabar pressures which will be beneficial to the optimum design of geometry and loading conditions in the experiment.
\end{abstract}

\footnotetext{
${ }^{*}$ Corresponding author.

Email: vlevitas@iastate.edu
} 
Keywords: Large elastic and plastic deformations, Static megabar pressures, Rotational diamond anvil cells, Finite element method.

\section{Introduction}

In high pressure research, static pressures up to several megabars are typically generated by compression of a thin sample sandwiched between two diamond anvils in a diamond anvil cell (DAC), see Fig. 1. Pressure generation is accompanied by very large plastic deformations of the sample as well as finite elastic deformations of the sample and diamond. One of the relevant directions in high pressure research is studying elastic and plastic properties of materials under pressure (Chen et al., 2012; Hemley et al., 1997; Jeanloz et al., 1991; Merkel et al., 2006; Singh et al., 2012; Weir et al., 1998). Other directions include studying high-pressure phase transformation (PT) and chemical reactions, studying various properties of high pressure phases, and searching for new phases (Drozdov et al, 2015; Toryan et al, 2016; Kaercher et al, 2012; Merkel et al, 2013; Wang et al., 2015; Zhao and Zhang, 2007). Applications include the geophysical studying of material behavior in Earth and other planets as well as material synthesis. One main goal is to either increase the level of maximum achievable pressure or achieve very high pressure for multiple times, both without breaking anvils. Recently, static pressure above $600 \mathrm{GPa}$ has been achieved (Dubrovinsky et al., 2012, 2015) using a special two-stage design.

One key point in understanding principles of producing high pressure, analyses of experimental data, and design of geometric and loading conditions is knowledge of stress-strain states of a sample, gasket (if used), and diamond anvils. A recent paper by Feng et al., 2016, in which the most advanced model and simulation of plastic flow and stress-strain states of a sample and anvils up to $300 \mathrm{GPa}$ are presented, contains quite a detailed review of corresponding literature for a DAC which will not be repeated here. In Feng et al., 2016, a thermodynamically consistent system 
of equations for large elastic and plastic deformations of an isotropic material is formulated for the sample, and the finite-strain third-order elasticity law for cubic crystals is utilized for diamond. Further, good correspondences between numerical data (Feng et al., 2016) and experiments (Hemley et al., 1997) are obtained in terms of pressure distributions at the contact surface, the sample thickness under pressure around $300 \mathrm{GPa}$, and the cupping-type shape of a sample due to deformation of the diamond anvils.

More complex loading, torsion under fixed applied force, and consequently, high pressure are utilized in pioneering Bridgman work on rotational Bridgman anvils made of hard alloys (Bridgman 1935 and 1952). Work with rotational Bridgman anvils includes studying the effect of plastic shear on various PTs and chemical reactions (Bridgman 1937 and 1947; Enikolopyan 1985 and 1989; Zharov, 1994) as well as determining pressure dependence of yield strength (Bridgman 1935 and 1952; Vereschagin et al., 1971). With the invention of the rotational diamond anvil cell (RDAC) (Alexandrova et al., 1993; Blank et al., 1984; Blank and Estrin, 2014), more advanced measurements became available, and thus more quantitative results have been obtained. Superposition of a large plastic shear on high pressure during torsion under fixed force leads to a number of exciting phenomena: (a) a significant reduction in phase transformation (PT) pressure by a factor of 2-5 (Alexandrova et al., 1993; Blank et al., 1984; Blank and Estrin, 2014; Levitas, 2004a; Novikov et al., 1999) and sometimes even of 10 (Ji et al., 2012); (b) an appearance of new phases which would not be obtained without shear straining (Blank and Estrin, 2014; Levitas et al., 2012; Novikov et al., 1999); (c) fast strain-controlled kinetics in which strain, instead of time, plays a role of a time-like parameter ( Blank and Estrin 2014; Levitas, 2004a and 2004b; Levitas and Zarechnyy, 2006); (d) Substitution of the reversible PT with an irreversible one (Blank and Estrin, 2014; Levitas et al., 2006), and (e) formation of nanograin and amorphous materials (Blank 
and Estrin, 2014; Levitas et al., 2012). Work with rotational Bridgman anvils recently finds new applications for producing nanograin materials and some PTs by high pressure torsion (HPT), see, e.g., Borodachenkova et al, 2015; Edalati and Horita, 2016; Kawasaki and Langdon, 2014; PerezPrado and Zhilyaev, 2009; Srinivasarao et al., 2013; Valiev et al., 2016a and 2016b. Reviews of some results on pressure and shear in a RDAC and HPT can be found in (Kawasaki and Langdon, 2014; Blank and Estrin, 2014; Edalati and Horita, 2016; Levitas, 2004b; Valiev et al., 2016a and 2016b). There are some recent studies on modeling and simulation of plastic flow (Levitas and Zarechnyy, 2010b) and strain-induced PTs in a RDAC (Feng and Levitas, 2013; Feng and Levitas, 2016; Feng et al., 2014; Levitas and Zarechnyy, 2010a), where assumptions of small elastic strains in the sample, pressure-independent yield strength, and rigid diamond were utilized. There are also FEM simulations of HPT (e.g. (Beygelzimer et al., 2016; Edalati et al., 2016; Figueiredo et al., 2011; Kim, 2001; Kim et al., 2003; Yoon et al., 2008)) for rigid-plastic materials and pressureindependent yield strength. These assumptions make the models unsuitable for processes in a RDAC under megabar pressures.

Rhenium is a silvery-white, heavy, group-VII transition metal, which has an HCP structure. Due to its large shear $(G)$ and bulk $(K)$ moduli and high compressive strength (Jeanloz et al., 1991; Manghnani et al., 1974; Vohra et al., 1987), rhenium is broadly used as a gasket material in DAC and RDAC at multi-megabar pressures. When annealed, this metal is ductile and can be bent, coiled, or rolled (Hammond, 2004). At high pressure, even strain-hardened rhenium can be plastically deformed by thousands of percent. A rhenium gasket is usually made of either polycrystal or compacted powder. The grain size of rhenium at a pressure of $250 \mathrm{GPa}$ (i.e., after large plastic deformation) is 10-20 nm (Singh et al., 2012), which is much smaller than the sample thickness $\left(1.8^{\mu \mathrm{m}}\right)$ at the same pressure. While some texture and strain-induced anisotropy are 
possible, it is shown in Levitas (1996) for more than 60 materials that starting with some strain and for monotonous loading (i.e., for a deformation path without sharp changes in directions) the initially isotropic polycrystalline materials are deformed as perfectly plastic and isotropic with a strain-history-independent limiting surface of the perfect plasticity. Such a model aptly described experiments from Hemley et al. (1997), see Feng et al. (1996). In this paper, we report the first modeling results for plastic flow and evolution of the stress-strain state for a rhenium sample compressed and twisted in RDAC under megabar pressures. We utilize the fully large deformation framework (in which both elastic and plastic strains, and rotation can be large) and finite element algorithms developed in Feng et al., 2016. This is quite a complex three-dimensional problem which, in addition to physical and geometric nonlinearities and pressure dependence of elastic and plastic properties, also involves nonlinearity due to contact sliding between sample and anvil. Due to three-dimensional character and two-dimensional contact sliding, this problem is much more sophisticated than the axisymmetric problem with one-dimensional radial sliding for compression in a DAC in Feng et al., 2016. In particular, this work represents verification of the effectiveness of the model and especially a numerical algorithm developed in Feng et al., 2016 for a more general case. In addition to quantitative information about evolution of the stress-strain fields, the following qualitative results are obtained. For the same axial force, thickness of the sample after rotation in a RDAC is much smaller than for compression in DAC. This is because frictional shear stress has the same magnitude for both cases, but for a RDAC it is inclined to the radial direction and the radial component of the friction stress is smaller than for a DAC. In fact, the sample thickness reduces by a factor of 3 after compression and by a factor of 30 during an anvil rotation of 1.6 radians. Next, even a slightly beveled $\left(8.5^{\circ}\right)$ contact surface of an anvil and sample makes a significant difference when very small thickness at the center and, consequently, a large gradient 
of thickness are achieved. In this case, a huge pressure gradient is found near the center in a RDAC, a phenomenon called here pressure self-focusing effect. Traditionally, plasticity relaxes stresses and reduces stress concentration. Here, the opposite effect happens. This effect can be used for significant increase in the maximum achievable pressure in the central region of a sample. Change in pressure away from the center is quite small. Since high pressure is localized in a very narrow region both in a sample and an anvil, there are much smaller chances to meet a critical defect which will cause the anvil to fracture. Due to the same reason, deformation and bending of an anvil are much smaller for a RDAC than for a DAC. In a DAC, bending of an anvil causes a cup-like shape of the contact surface between the sample and anvil (cupping phenomenon) which arrests radial plastic flow and effectively does not allow pressure to increase. In a RDAC, cupping was not observed up to $240 \mathrm{GPa}$, and there was just a small reduction in the beveling angle. Thus, rotation of an anvil is not only a way to superpose plastic shear to run PTs and chemical reactions, but it is also an effective way to increase maximum achievable pressure, albeit, in a small volume. Counterintuitively, increasing yield strength, pressure dependence of the yield strength, and the friction coefficient reduce the pressure gradient and maximum pressure when compared at the same force. Also, we found the traditional method for determining pressure dependence of the yield strength based on measured force and torque (Levitas, 2004a; Levitas et al., 2006; Manghnani et al., 1974) may have very large errors. In particular, torque is not constant and increases up to a rotation of 1.6 radians for a perfectly plastic material due to continuous redistribution between radial and circumferential friction stresses. Thus, constant torque cannot be interpreted as a lack of strain hardening. Also, due to Coulomb friction, the magnitude of shear friction stress is smaller than the yield strength in shear under the given pressure. 
This paper is organized as follows. Complete system of equations and material parameters are presented in Section 2. Geometry and problem formulation are given in Section 3. Main results for sample and anvils are presented in Sections 4 and 5, respectively. Effects of material parameters, friction coefficients, axial load, and geometry of an anvil are elaborated in Section 6. Section 7 contains concluding remarks.

\section{A complete system of equations and material parameters}

The contractions of second-order tensors $\boldsymbol{A}$ and $\boldsymbol{B}$, and the fourth-order tensors over one and two indices are defined as $\boldsymbol{A} \cdot \boldsymbol{B}=\left\{A_{i j} B_{j k}\right\}$ and $\boldsymbol{A}: \boldsymbol{B}=\left\{A_{i j} B_{j i}\right\}$ as well as $\boldsymbol{A} \cdot \boldsymbol{B}=\left\{A_{i j k m} B_{m n l q}\right\}$

and $\boldsymbol{A}: \boldsymbol{B}=\left\{A_{i j k m} B_{m k l q}\right\}$, respectively. The subscript $s$ stands for symmetrization, the superscripts -1 and $T$ are for the inversed and transposed tensors, $\boldsymbol{I}$ is the second-order unit tensor, det means the determinant, and the subscripts $e$ and $p$ designate elastic and plastic deformation gradient or strains. Detailed derivations of the equations can be found in Levitas, 1996 and Feng, et al., 2016. Rate-independent, isotropic, and perfectly plastic material is considered.

\section{Nonlinear elastoplasticity for the sample}

The deformation gradient

$$
\boldsymbol{F}=\frac{\partial \boldsymbol{r}}{\partial \boldsymbol{r}_{0}}=\boldsymbol{F}_{e} \cdot \boldsymbol{F}_{p}=\boldsymbol{R}_{e} \cdot \boldsymbol{U}_{e} \cdot \boldsymbol{R}_{p} \cdot \boldsymbol{U}_{p}=\boldsymbol{R}_{e} \cdot \boldsymbol{U}_{e} \cdot \boldsymbol{U}_{p}=\boldsymbol{V}_{e} \cdot \boldsymbol{R}_{e} \cdot \boldsymbol{U}_{p}\left(\boldsymbol{R}_{p}=\boldsymbol{I} ; \boldsymbol{V}_{e}=\boldsymbol{R}_{e} \cdot \boldsymbol{U}_{e} \cdot \boldsymbol{R}_{e}^{T}\right)
$$

is decomposed into elastic $\boldsymbol{F}_{e}$ and plastic $\boldsymbol{F}_{p}$ parts. $\boldsymbol{R}_{e}$ and $\boldsymbol{R}_{p}$ are the proper orthogonal elastic and plastic rotation tensors. $\boldsymbol{U}_{e}$ and $\boldsymbol{U}_{p}$ are the symmetric elastic and plastic right stretch tensors, and $V_{e}$ is the elastic left stretch tensor.

Eulerian and Lagrangian elastic strain tensors are

$$
\boldsymbol{B}_{e}=0.5\left(\boldsymbol{V}_{e} \cdot \boldsymbol{V}_{e}-\boldsymbol{I}\right) ; \quad \boldsymbol{E}_{e}=0.5\left(\boldsymbol{U}_{e} \cdot \boldsymbol{U}_{e}-\boldsymbol{I}\right) .
$$


Decomposition (1) for isotropic materials can be transformed by the following decomposition of the deformation rate $d$ :

$$
\boldsymbol{d}=\stackrel{\nabla}{\boldsymbol{B}}_{e}-2\left(\boldsymbol{d} \cdot \boldsymbol{B}_{e}\right)_{s}+\boldsymbol{V}_{e} \cdot \boldsymbol{D}_{p} \cdot \boldsymbol{V}_{e} ; \boldsymbol{D}_{p}=\boldsymbol{R}_{e} \cdot\left(\dot{\boldsymbol{U}}_{p} \cdot \boldsymbol{U}_{p}^{-1}\right)_{s} \cdot \boldsymbol{R}_{e}^{t} ; \quad \stackrel{\nabla}{\boldsymbol{B}}_{e}=\dot{\boldsymbol{B}}_{e}-2\left(\boldsymbol{W} \cdot \boldsymbol{B}_{e}\right)_{s},
$$

where $\boldsymbol{D}_{p}$ is the plastic deformation rate, $\boldsymbol{W}$ is the spin tensor in the current configuration, and $\stackrel{\nabla}{B}_{e}$ is the Jaumann objective time derivative.

The nonlinear elastic constitutive equation for an isotropic material is

$$
\boldsymbol{\sigma}=\frac{1}{\operatorname{det} \boldsymbol{F}}\left(2 \boldsymbol{B}_{e}+\boldsymbol{I}\right) \cdot \frac{\partial \Psi}{\partial \boldsymbol{B}_{e}}
$$

where $\sigma$ is Cauchy stress, and $\Psi$ is the third-order Murnaghan potential:

$$
\Psi\left(\boldsymbol{B}_{e}\right)=\frac{\lambda_{e}+2 G}{2} I_{1}^{2}-2 G I_{2}+\left(\frac{l+2 m}{3} I_{1}^{3}-2 m I_{1} I_{2}+n I_{3}\right)
$$

Here $\lambda_{e}, G, m, l$, and $n$ are second- and third-order elastic constants, and $I_{i}$ is the $i^{t h}$ invariant of the strain tensor $\boldsymbol{B}_{e}$ :

$$
I_{1}=B_{e_{11}}+B_{e_{22}}+B_{e_{33}} ; \quad I_{2}=B_{e_{22}} B_{e_{33}}-B_{e_{23}}^{2}+B_{e_{11}} B_{e_{33}}-B_{e_{13}}^{2}+B_{e_{22}} B_{e_{11}}-B_{e_{12}}^{2} ; I_{3}=\operatorname{det} \boldsymbol{B}_{e} .
$$

The pressure-dependent $\mathrm{J}_{2}$ yield condition is utilized.

$$
\varphi=\sqrt{3 / 2 s: s}-\sigma_{y}=0
$$

where $s$ is the deviatoric part of Cauchy stress, and $\sigma_{y}$ is the yield strength which linearly

depends on pressure $p$ :

$$
\sigma_{y}(p)=\sigma_{y 0}+b p
$$

The plastic flow rule is

$$
D_{p}=R_{e} \cdot\left(\dot{U}_{p} \cdot U_{p}^{-1}\right)_{s} \cdot R_{e}^{t}=\lambda s / \sqrt{s: s}
$$

where $\lambda \geq 0$ is a scalar function determined from the consistency condition $\dot{\varphi}=0$. Thus, $\lambda=0$ in the elastic region $(\varphi(s, p)<0$ or $\varphi(s, p)=0$, but $\dot{\varphi}(s, p)<0)$ and $\lambda>0$ in the elastoplastic region $(\varphi(s, p)=0$ and $\dot{\varphi}(s, p) \geq 0)$.

Accumulated plastic strain is defined as 


$$
\dot{q}=\left(2 \boldsymbol{D}_{p}: \boldsymbol{D}_{p} / 3\right)^{0.5}=\sqrt{2 / 3} \lambda
$$

While $q$ does not appear in any of our equations, it is used to represent the results on the equivalent plastic strain obtained in our simulations.

Equilibrium equations in the deformed configuration is:

$$
\nabla \cdot \boldsymbol{\sigma}=\mathbf{0}
$$

Elastic constants (Feng et al., 2016; Lv et al., 2012; Manghnan et al., 1974) are $K=380 \mathrm{GPa}, G=200 \mathrm{GPa}, \lambda_{e}=247 \mathrm{GPa}, l=-291 \mathrm{GPa}, m=-662 \mathrm{GPa}$, and $n=0$.

The initial yield strength and its pressure derivative are (Feng et al., 2016, Jeanloz et al., 1991) $\sigma_{y 0}=8.00 \mathrm{GPa}$ and $b=0.04$.

\section{Nonlinear anisotropic elasticity for single-crystal diamond}

Elastic deformations of diamond will only be considered, and the subscript $e$ will be omitted. For anisotropic materials, the elasticity law is

$$
\boldsymbol{\sigma}=\boldsymbol{F} \cdot \tilde{\boldsymbol{T}}(\boldsymbol{E}) \cdot \boldsymbol{F}^{T} / \operatorname{det} \boldsymbol{F} ; \quad \tilde{\boldsymbol{T}}=\partial \Psi / \partial \boldsymbol{E},
$$

where $\tilde{\boldsymbol{T}}$ is the second Piola-Kirchhoff stress. The third-order elastic energy $\Psi$ for cubic symmetry is as follows:

$$
\begin{aligned}
& \Psi=0.5 c_{11}\left(\eta_{1}^{2}+\eta_{2}^{2}+\eta_{3}^{2}\right)+c_{12}\left(\eta_{1} \eta_{2}+\eta_{1} \eta_{3}+\eta_{2} \eta_{3}\right)+0.5 c_{44}\left(\eta_{4}^{2}+\eta_{5}^{2}+\eta_{6}^{2}\right)+c_{111}\left(\eta_{1}^{3}+\eta_{2}^{3}+\eta_{3}^{3}\right) / 6 \\
& +0.5 c_{112}\left[\eta_{1}^{2}\left(\eta_{2}+\eta_{3}\right)+\eta_{2}^{2}\left(\eta_{1}+\eta_{3}\right)+\eta_{3}^{2}\left(\eta_{1}+\eta_{2}\right)\right]+c_{123} \eta_{1} \eta_{2} \eta_{3}+0.5 c_{144}\left(\eta_{1} \eta_{4}^{2}+\eta_{2} \eta_{5}^{2}+\eta_{3} \eta_{6}^{2}\right) \\
& +0.5 c_{166}\left[\left(\eta_{2}+\eta_{3}\right) \eta_{4}^{2}+\left(\eta_{1}+\eta_{3}\right) \eta_{5}^{2}+\left(\eta_{1}+\eta_{2}\right) \eta_{6}^{2}\right]+c_{456} \eta_{4} \eta_{5} \eta_{6}
\end{aligned}
$$

where $\eta_{1}=E_{11}, \eta_{2}=E_{22}, \eta_{3}=E_{33}, \eta_{4}=2 E_{23}, \eta_{5}=2 E_{31}$, and $\eta_{6}=2 E_{12}$.

In this paper, the second-order elastic constants used are from Nielsen, 1986:

$$
c_{11}=1050 \mathrm{GPa}, c_{12}=127 \mathrm{GPa} \text {, and } c_{44}=550 \mathrm{GPa},
$$

and the third-order elastic constants are as in Lang and Gupta, 2011:

$$
c_{111}=-7603 \mathrm{GPa}, c_{112}=-1909 \mathrm{GPa}, c_{123}=-835 \mathrm{GPa},
$$




$$
c_{144}=1438 \mathrm{GPa}, c_{166}=-3938 \mathrm{GPa}, c_{456}=-2316 \mathrm{GPa} .
$$

\section{Geometries and boundary conditions}

A schematic of a RDAC is shown in Fig. 1 (a), where the sample is sandwiched between two diamond anvils, and the geometry of the anvils as well as the sample is axisymmetric. Different from a DAC, the loads in the RDAC are three-dimensional and followed by two steps. First, a normal stress $\sigma_{n}$ is applied to the diamond anvils Second, one of anvils is rotated with respect to the other by a rotation angle $\varphi$ under a fixed $\sigma_{n}$. While the material of a single crystal with a cubic atomic structure is usually not axisymmetric, the anisotropy of diamond along the circumferential direction is negligible due to $\left(c_{11}-c_{12}\right) /\left(2 c_{44}\right) \approx 1$. In particular, in Hemley et al., 1997, the 3D experimental image of a sample's thickness is practically axisymmetric. Also, it is assumed a conventional diamond cell is axisymmetric typically with the 001 orientation along the $z$ axis. Due

to symmetry, the results will be discussed using a quarter of a DAC as shown in Fig. 1. The boundary conditions are as follows:

(1) The normal stress $\sigma_{n}$ is applied at the top surface $A B$ of the anvil. During rotation, the torsion is applied to the top surface of the anvil under a constant normal stress $\sigma_{n}$.

(2) The radial displacement $u_{r}$ and shear stresses $\tau_{r z}$ and $\tau_{z \theta}$ are zero at the symmetry axis $r=0$ (the lines $A D$ and $C D$ for the anvil and the sample, respectively).

(3) At the contact surface (the line $D G E$ ), the Coulomb friction model is used and the details will be introduced below.

(4) At the symmetry plane $z=0$ (the line $C H$ ), the radial shear stress $\tau_{r z}=0$. The circumferential and axial displacements are $u_{\theta}=u_{z}=0$, respectively.

(5) Other surfaces not mentioned above are stress-free. 


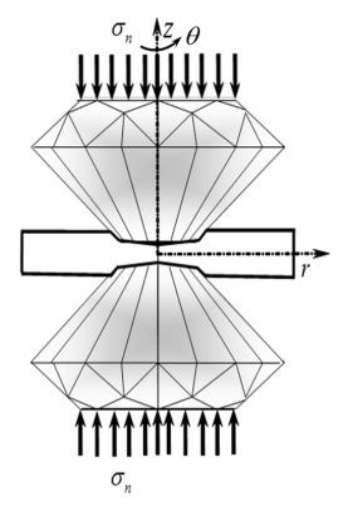

(a)

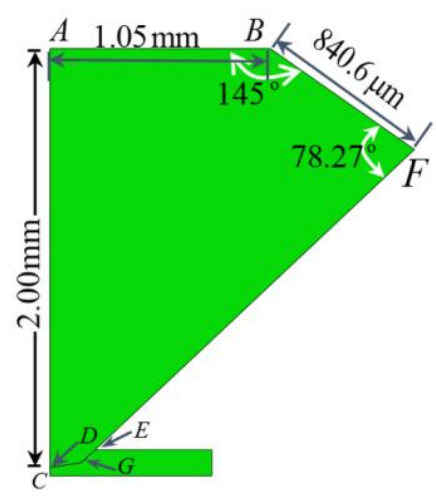

(b)

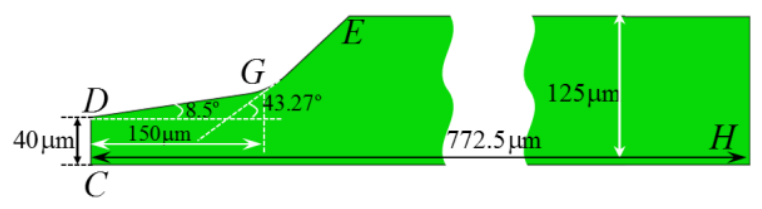

(c)

FIG. 1. (a) Rotational diamond anvil cell scheme, (b) a quarter of the sample and anvil in the initial undeformed state as well as geometry of the anvil, and (c) the geometry of sample.

In a RDAC, the friction stress $\boldsymbol{\tau}$ is a vector inclined to the radial direction in contrast to loading in a DAC. As shown in Fig. 4a, the friction stress vector $\boldsymbol{\tau}$ can be decomposed into two orthogonal components $\tau_{1}$ and $\tau_{2}$ along the contact surface. $\tau_{2}$ is along the circumferential (torsional) direction $\theta$, and $\tau_{1}$ is the component of the friction stress $\boldsymbol{\tau}$ perpendicular to $\tau_{2}$. While $\tau_{2}$ is not along the $r$ direction, it intersects with the $z$ axis and projects on the $C H$ plane in Fig. 1c along the $r$ direction. If the contact surface is flat (i.e. parallel to the $C H$ plane), $\tau_{1}=\tau_{r z}$ and $\tau_{2}=\tau_{\theta z}$, which was the case in (Feng and Levitas, 2013, Feng and Levitas, 2016, Feng, et al., 2014, Levitas and Zarechnyy, 2010). In the current paper, $\tau_{1} \neq \tau_{r z}$ and $\tau_{2} \neq \tau_{\theta z}$ at each contact point because the contact surface is inclined. Note, the classic Coulomb friction model is used. When the magnitude of friction stress $|\boldsymbol{\tau}|=\sqrt{\tau_{1}^{2}+\tau_{2}^{2}}$ reaches $\mu \sigma_{c}$, slipping is allowed ( $\sigma_{c}$ is the normal contact stress at the contact surface between the diamond and the sample) and the direction of slippage vector $s$ is along the direction of friction stress $\tau$, i.e., $\frac{s}{|s|}=\frac{\tau}{|\tau|}$. Otherwise $\left(|\boldsymbol{\tau}|<\mu \sigma_{c}\right)$, the cohesion condition (continuity of displacements) is applied. The friction 
coefficient is accepted to be $\mu=0.1$, which is the value that gives the best correspondence between simulations and experiments up to $285 \mathrm{GPa}$ in Feng, et al., 2016.

In a RDAC, during the rotation of the anvil under a fixed applied normal stress $\sigma_{n}$, the sample material flows from the center to the periphery which can cause significant thickness reduction and larger plastic deformation than in a DAC. Consequently, a very fine mesh in the region with large deformation should be assigned. For example, at the center $(r=0)$, there are 100 elements along half the sample thickness as shown in Fig. 2. Even when the number of elements is decreased by a factor of two, the same results are obtained for all stresses. This proves the obtained solutions are mesh independent.
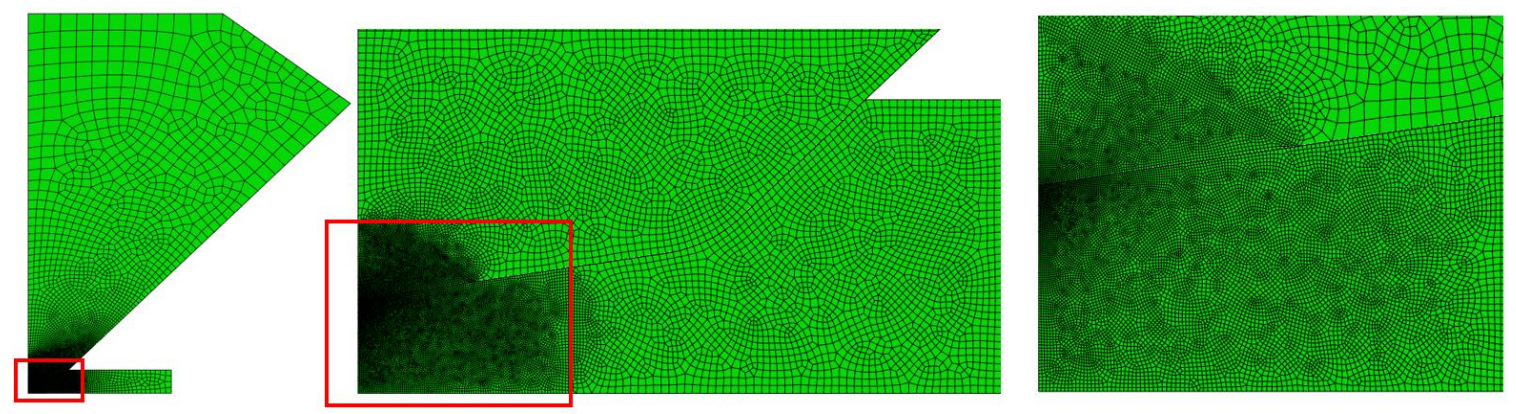

FIG. 2 Mesh distribution in simulations. The zoomed part is shown to the right of the zooming box. At $r=0$ in sample, there are 100 elements along the thickness direction.

\section{Evolution of stress-strain fields in a sample in a RDAC}

In this section, stress and strain fields in a sample during rotation of one anvil with respect to another under a fixed applied normal stress $\sigma_{n}=1.1 \mathrm{GPa}$ will be discussed. Fig. 3 (a) shows for the rotation angle $\varphi=0$ and $\sigma_{n}=1.1 \mathrm{GPa}$, the maximum pressure $\left(p_{\max }=44.8 \mathrm{GPa}\right)$ in the sample is at the center, the pressure gradient is small in the entire sample, and the thickness at the center is reduced around three times the initial undeformed configuration. With the growth of 
rotation angle $\varphi$ from 0 to 1.6 radians, the material flows from the center to the periphery and the thickness reduces significantly. At $\varphi=1.6$, the thickness at the center of the sample is reduced by about 90 times from the initial $80 \mu \mathrm{m}$ to $0.9 \mu \mathrm{m}$. From Fig. 3 (a), there is a very large thickness gradient along the radial direction at $\varphi=1.6$, and the thickness increased from $0.9 \mu \mathrm{m}$ at the $r=0$ $\mu \mathrm{m}$ to $30 \mu \mathrm{m}$ at $r=120 \mu \mathrm{m}$, while the shape of the contact surface does not vary significantly. During the rotation, except for the region at the center, the pressure does not change significantly due to a fixed normal stress $\sigma_{n}$, and the pressure gradient is not large along the thickness direction. However, at the center a very large pressure gradient and, consequently, pressure unexpectedly appear. This phenomenon is called here the pressure self-focusing effect. It is unexpected because it was never observed in experiments or found theoretically. Indeed, for flat anvils and without PTs, it was obtained experimentally (Blank et al., 1984; Blank and Estrin, 2014; Novikov et al., 1999) analytically (Levitas, 2004a and 2004b), and in FEM modeling (Levitas and Zarechnyy, 2010b) that the pressure distribution does not vary during torsion under a constant force. However, beveled anvils and very small thicknesses were not studied experimentally nor theoretically.

Fig. 3 (b) shows the pressure distribution of RDAC and DAC at the same maximum pressure of $175 \mathrm{GPa}$ at the center. The pressure is larger in a DAC than a RDAC everywhere except the center. The reason is that in a DAC the only way to increase the pressure in the sample is through enlarging the applied normal stress $\sigma_{n}$ which leads to a pressure growth in the entire sample. At the same time, the pressure grows in a RDAC through rotation at a smaller constant applied normal stress $\sigma_{n}$. The RDAC provides a better way to obtain megabar pressures than the DAC. For the same maximum pressure, the contact pressure in the rest of the contact surface in a DAC is much larger than in a RDAC. This causes bending of the anvil and the cupping 
phenomenon (i.e., the slope of contact surface is getting negative at the periphery). When cupping occurs, radial plastic flow is suppressed, thickness does not reduce further, and the maximum pressure essentially does not grow. In a RDAC, the deformation of the anvil is reduced in the major external part of the surface which postpones the cupping phenomenon to much higher pressures. It is clear from Fig. 3 (a) that the shape of the contact surface almost does not change at the periphery.

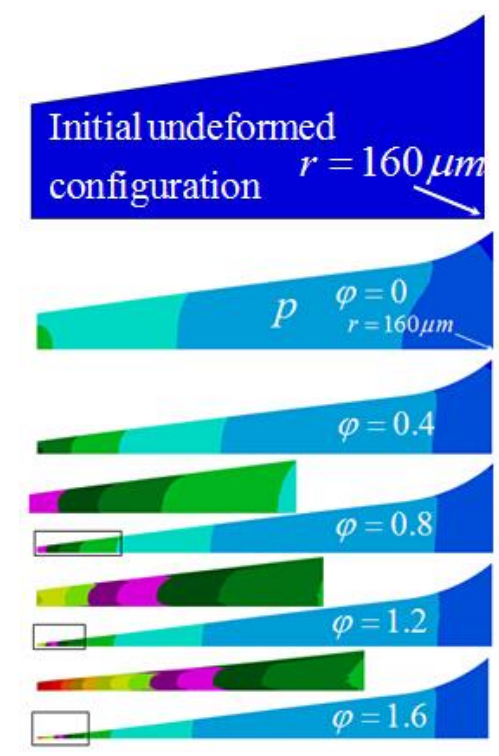

(a)

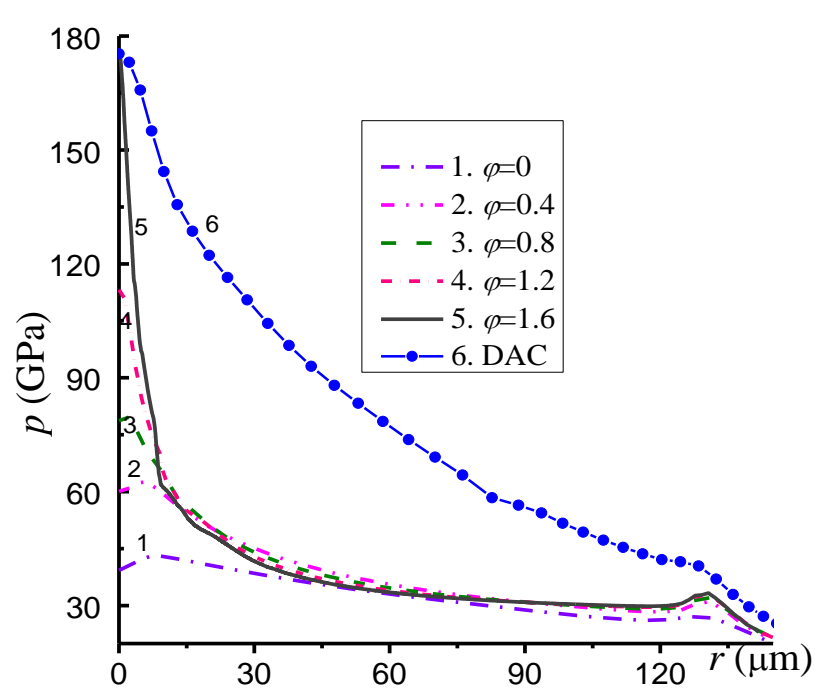

(b)

FIG. 3. Distribution of pressure $p$ in the sample $(0 \leq r \leq 160 \mu \mathrm{m})$ (a) and at the contact surface (b) under a fixed applied normal stress $\sigma_{n}=1.1 \mathrm{GPa}$ with growing rotation angle $\varphi: 0,0.4,0.8$, 1.2 and 1.6. The zoomed central part of the sample is shown above the sample.

To understand the reason for the sharp increase in pressure in a RDAC in Fig. 3(a), we consider equilibrium of an infinitesimal element of the sample along the radial direction in the deformed configuration in a RDAC at an arbitrary position $r$ shown in Fig. 4(a). All stresses and geometric parameters are presented in Fig. 4(a), where normal stresses $\sigma_{r r}$ and $\sigma_{\theta \theta}$ are averaged over the sample thickness. Summation of all forces in the $r$ direction yields 


$$
\begin{aligned}
& \sigma_{r r} r h \cdot \mathrm{d} \theta-\left(\sigma_{r r}+\mathrm{d} \sigma_{r r}\right)(r+\mathrm{d} r)(h+2 \cdot \mathrm{d} r \cdot \tan \alpha) \mathrm{d} \theta-2 \tau_{1} r \cdot \cos \alpha \cdot \mathrm{d} \theta \frac{\mathrm{d} r}{\cos \alpha} \\
& +2 \sigma_{c} r \cdot \sin \alpha \cdot \mathrm{d} \theta \frac{\mathrm{d} r}{\cos \alpha}+2 \sigma_{\theta \theta} h \cdot \mathrm{d} r \cdot \frac{\mathrm{d} \theta}{2}=0 .
\end{aligned}
$$

After simple algebra and neglecting the higher order infinitesimal terms, Eq. (14) can be simplified into an equilibrium equation

$$
\frac{d \sigma_{r r}}{d r}=-\frac{2 \tau_{1}}{h}+\frac{2 \tan \alpha \cdot\left(\sigma_{c}-\sigma_{r r}\right)}{h}+\frac{\sigma_{\theta \theta}-\sigma_{r r}}{r} .
$$

Notice that circumferential friction $\tau_{2}$ is not involved in Eq. (15) since it is perpendicular to the $\mathrm{r}$ direction. An equilibrium equation in the circumferential direction is not informative because two $\tau_{2}$ values in opposite directions at the top and bottom surfaces shown in Fig. 4 (a) compensate each other.

For an intense plastic flow and full adhesion condition, the friction stress at the contact surface reaches the yield strength in shear $\tau_{y}=\sigma_{y} / \sqrt{3}$ (where $\sigma_{y}$ is the yield strength in compression), which leads to $\sigma_{z z}=\sigma_{r r}=\sigma_{\theta \theta}=p$ at the contact surface due to the von Mises yield condition. For Coulomb friction, the magnitude of the friction stress is $\mu \sigma_{c}$. Since the sample is thin and the variation of normal stress along the thickness is small (Feng, et al., 2016), we obtain for averaged stresses $\sigma_{z z} \approx \sigma_{r r} \approx \sigma_{\theta \theta} \approx p$. If $\alpha$ is a small angle, like $\alpha=8.5^{\mathrm{o}}$ in Fig. 1, then $\sigma_{c} \approx \sigma_{z z} \approx \sigma_{r r} \approx p$ , and we obtain a simplified equilibrium equation

$$
\frac{d p}{d r}=\frac{d \sigma_{r r}}{d r}=-\frac{2 \tau_{1}}{h}=-\frac{2 \tau_{1}}{h_{0}+2 r \tan \alpha},
$$

which was used (mostly for $\alpha=0$ ) in previous works (Eremets, 1996; Thomsen, 1965; Feng and Levitas 2013; Feng and Levitas, 2016 and 2017; Feng et al., 2014; Levitas and Zarechnyy, 2010). 
Fig. 5 plots the corresponding evolution of distributions of the radial $\tau_{1}$ and circumferential $\tau_{2}$ components, resultant friction stresses $|\tau|$, and inverse sample thickness $1 / h$. Before rotation, pressure and the pressure gradient are small due to a small radial friction stress and a large sample thickness. During rotation of an anvil, the velocity of relative sliding and friction shear stress are inclined to the radial direction, and the radial component $\tau_{1}$ of friction stress is smaller than the total friction stress. This is true in regions with the magnitude of friction equal to Coulomb friction $\mu \sigma_{c}$ or with resultant friction stress equal to the yield strength in shear $\tau_{y}$. With increasing torsion, the torsional component of shear stress $\tau_{2}$ increases, and the radial component $\tau_{1}$ decreases in the major outer part of the sample, but both $\tau_{2}$ and $\tau_{1}$ increase in the central part due to an increase in yield strength during pressure rise. The increase in radial friction stress at the center slightly contributes to a large pressure gradient near the center, but is not the main reason for a sharp pressure gradient at the center of the sample. Fig. 5(d) shows the main reason for the pressure-self-focusing effect at the center which is a localized strong increase in the inverse thickness $1 / h$, to which the pressure gradient is proportional according to Eq. (16). Thus, for a flat anvil, the pressure self-focusing should not occur which agrees with experimental (Blank, 2014; Blank et al., 1984; Novikov et al., 1999), analytical (Levitas, 2004a and 2004b), and FEM (Levitas and Zarechnyy, 2010b) results. In a DAC, the thickness is much larger. Also, the only way to increase the pressure in the sample in a DAC is to increase the compressive load $\sigma_{n}$, which causes bending of the diamond contact surface. Both reasons significantly reduce $1 / h$ for DAC vs RDAC. Contact sliding occurs in the major external part of the sample, and the magnitude of the friction stress is equal to $\mu \sigma_{c}$, which is below the yield strength in shear for the corresponding pressure. The friction stress reaches the yield strength in shear in a small region near the center, 
which hardly contributes to the torque. In one of the traditional methods of determining the pressure dependence of the yield strength (Bridgman, 1935 and 1937; Vereschagin et al., 1971), pressure was estimated as force per unit anvil area, and the yield strength in shear was calculated assuming that $\tau_{2}=\tau_{y}$ is homogeneously distributed and can be easily estimated in terms of measured torque. As we can see, none of these assumptions are satisfactory: pressure is strongly heterogeneous, $\tau_{2}$ is only part of $|\tau|$ and is not homogeneous either, and even $|\tau|$ is smaller than $\tau_{y}$ due to Coulomb sliding. Torque versus rotation angle did not saturate even at $\varphi=1.6$ radians (Fig. 5 (e)). One may believe it is better to assume full adhesion or plastic friction $|\tau|=\tau_{y}$ instead of Coulomb sliding. However, good correspondence of our simulations with experiments for compression in DAC up to almost $300 \mathrm{GPa}$ in Feng et al., 2016 was obtained for Coulomb friction with the same coefficient $\mu=0.1$. Thus, at least at high pressures, the method for determining the pressure dependence of the yield strength in (Bridgman, 1935 and 1937; Vereschagin et al., 1971) is not reliable.

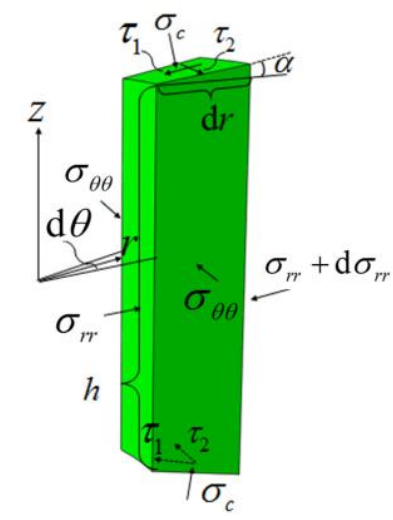

(a)

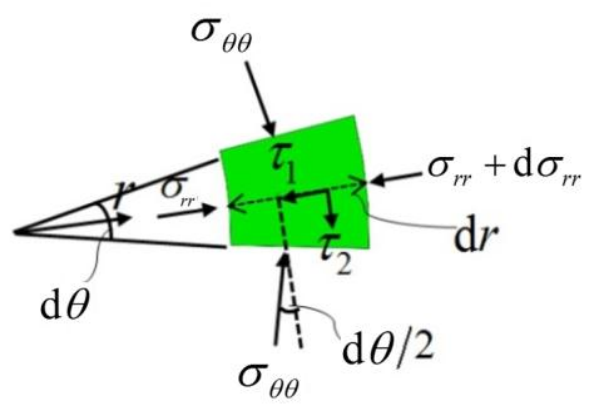

(b)

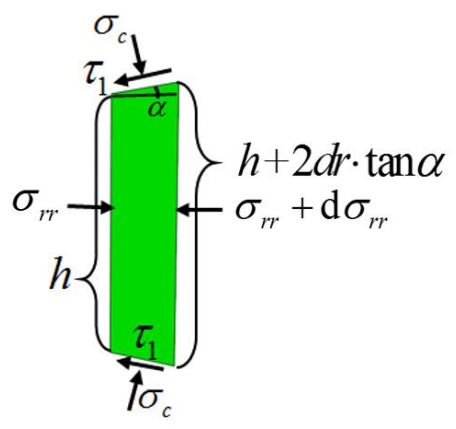

(c)

Fig. 4. Free-body diagram for derivation of the equilibrium condition in the radial direction for an infinitesimal element of the sample in 3D (a), and 2D projections (b) and (c). 


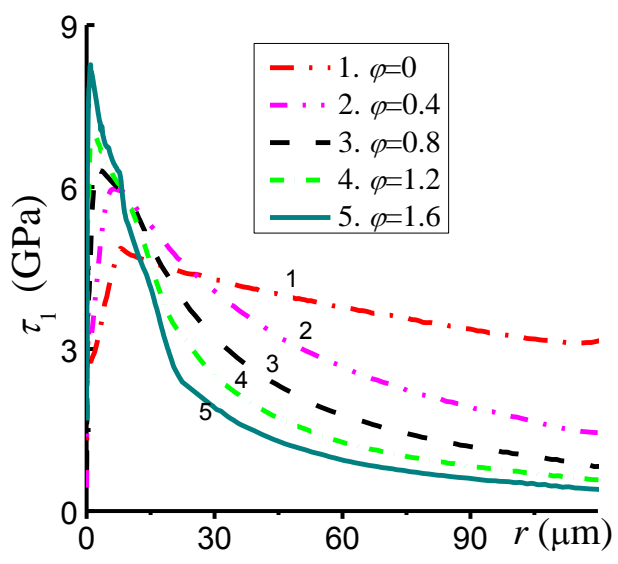

(a)

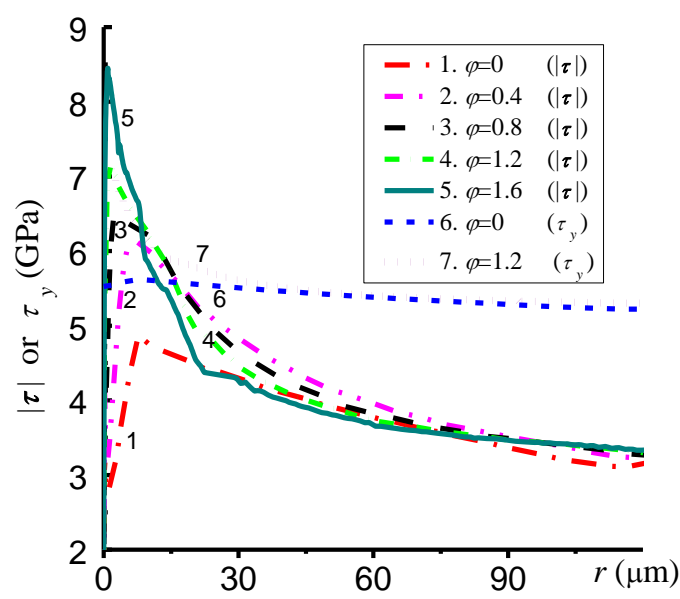

(c)

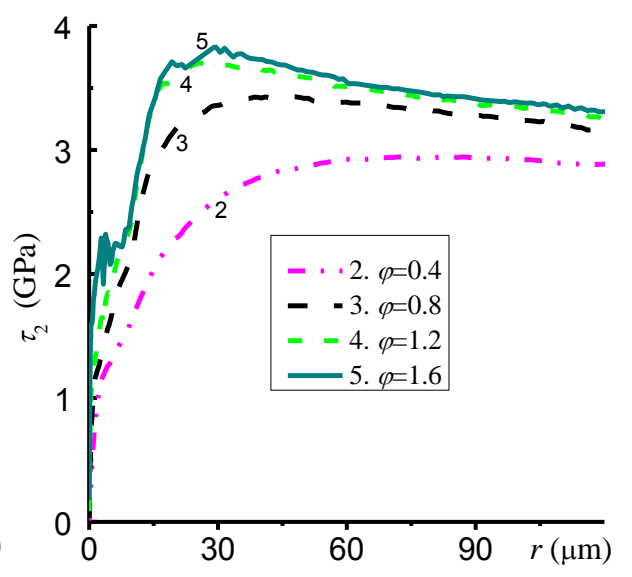

(b)

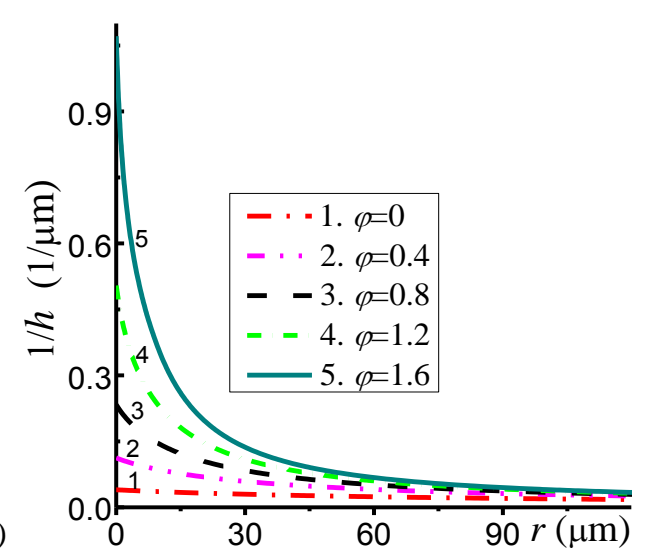

(d)

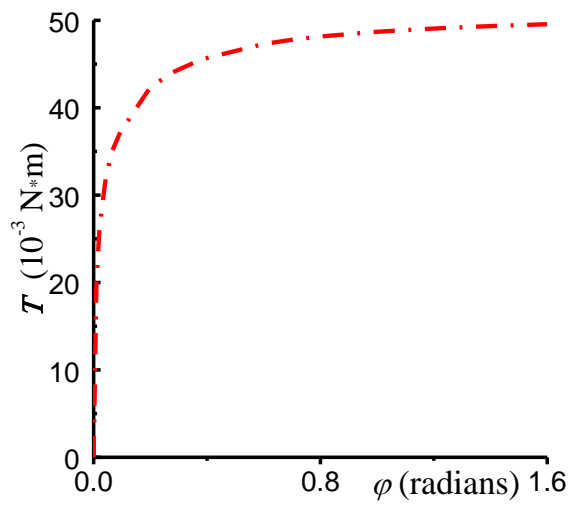

(e)

Fig. 5. Distributions of the friction stresses $\tau_{1}$ (a), $\tau_{2}$ (b), and $|\tau|$ or $\tau_{y}$ (c); inverse thickness $1 / h$ of sample (d), and applied torque $T$ on one of anvils (e) under a fixed applied normal stress $\sigma_{n}=1.1 \mathrm{GPa}$ with the increasing rotation angle $\varphi$. 


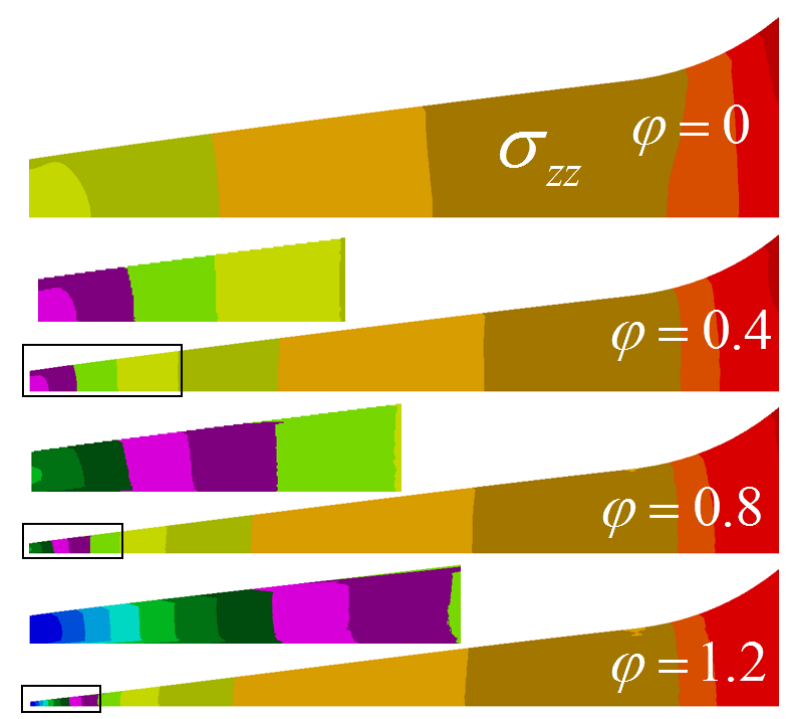

(a)

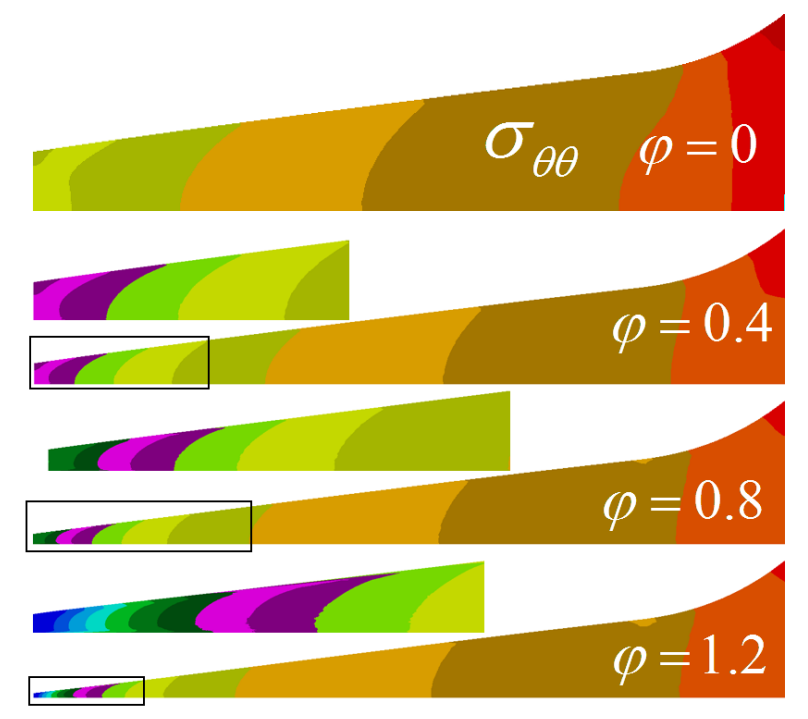

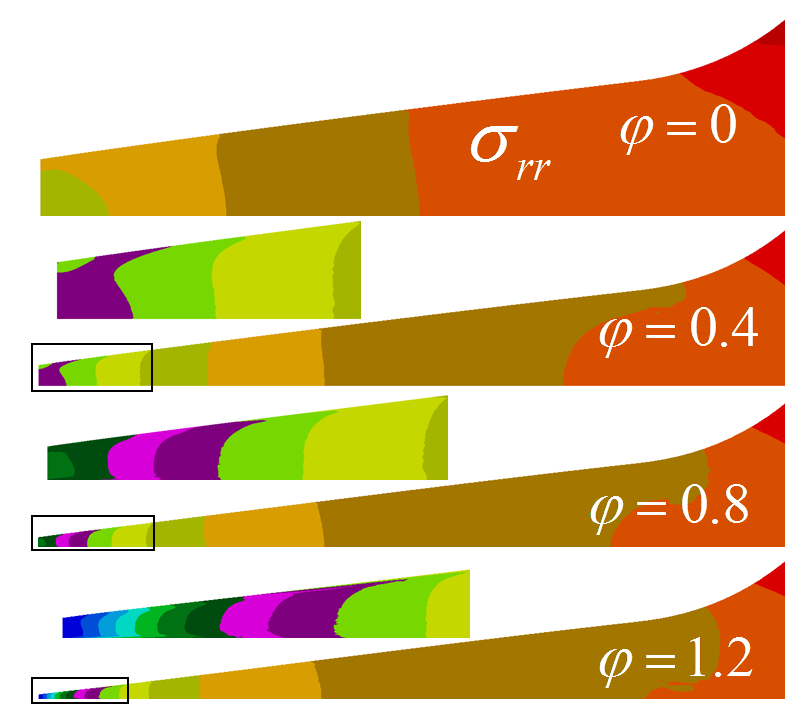

(b)

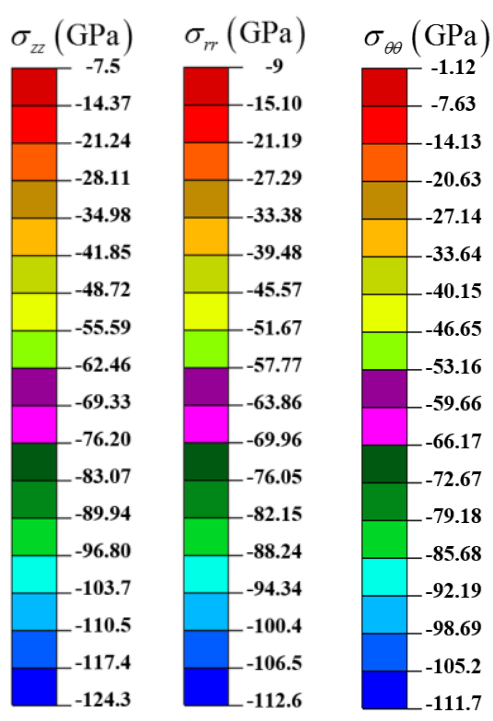

(c)

FIG. 6. Distributions of normal stresses $\sigma_{z z}$ (a), $\sigma_{r r}$ (b) and $\sigma_{\theta \theta}$ (c) in the sample $(0 \leq r \leq 160 \mu \mathrm{m})$ under a fixed applied normal stress $\sigma_{n}=1.1 \mathrm{GPa}$ with the growing rotation angle $\varphi$. The zoomed central part of a sample is shown above the sample.

The normal stress components $\sigma_{z z}, \sigma_{r r}$ and $\sigma_{\theta \theta}$ in the sample during the growth of rotation are shown in Fig. 6. All three normal stresses are distributed very similarly in the sample: their magnitudes are close because they are much larger than the yield strength; they are very large only in the central region of sample and are much smaller in the rest of a sample; the stress 
gradients are very small along the thickness but large along the radial direction, especially at the center of a sample. In the major part of a sample, the gradient of normal stress $\sigma_{z z}$ along the thickness is smaller, but is larger along the radial direction. Also, the magnitude of $\sigma_{z z}$ is also slightly larger in comparison with two other normal stresses, $\sigma_{r r}$ and $\sigma_{\theta \theta}$.

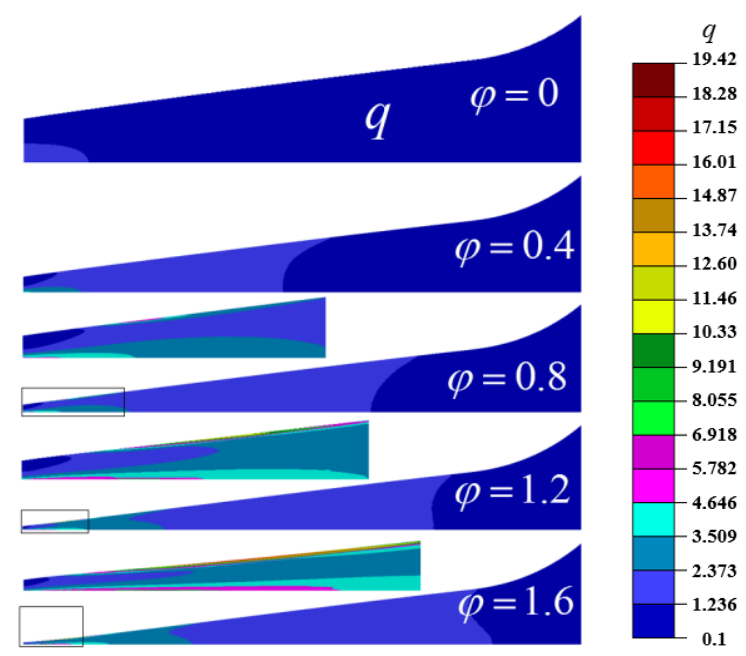

(a)

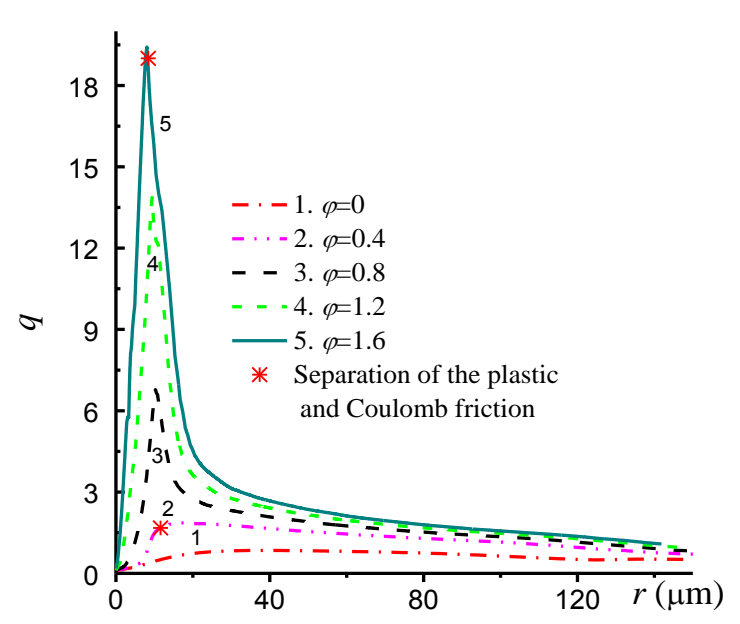

(b)

FIG. 7. Distributions of the accumulated plastic strain $q$ in the sample $(0 \leq r \leq 160 \mu \mathrm{m})$ (a) and at the contact surface (b) under a fixed applied normal stress $\sigma_{n}=1.1 \mathrm{GPa}$ with the rowing rotation angle $\varphi$.

Fig. 7 exhibits the evolution of the accumulated plastic strain, $q$, in the sample and at the contact surface during the growth of the rotation angle, $\varphi$. At the contact surface, the separation radii between the Coulomb friction $\left(\tau=\mu \sigma_{c}\right)$ and plastic friction $\left(\tau=\tau_{y}\right)$ are at $r=1.6 \mu \mathrm{m}$ and $r=$ $8.4 \mu \mathrm{m}$ for the rotation angles 0.4 and 1.6, respectively. Coulomb sliding occurs for larger radii, and at the central part of the contact surface the sample points move along with the diamond anvil. This causes a very large plastic strain near the surface due to material flow along the circumferential direction. At the center of sample $(r=0)$ there is no circumferential movement, so 
the plastic strain is small. Below the contact surface with this plastic friction, the materials can flow from the center to the periphery, which causes the thickness reduction. Close to the symmetry plane, radial plastic flow intensifies due to reduction of the effect of contact friction (Fig. 7(a)).

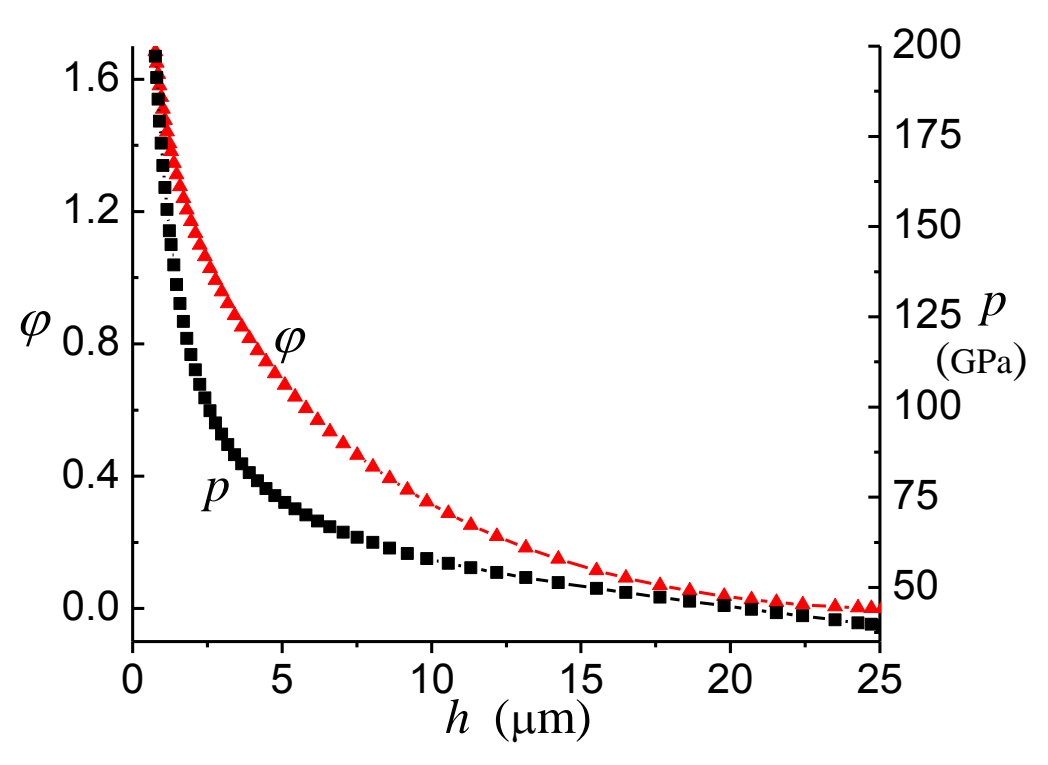

FIG. 8. The variation of the rotation angle and pressure at the contact surface at the center $(r=0)$ versus the thickness of the sample $h$ at $r=0$, under a fixed applied stress $\sigma_{n}=1.1 \mathrm{GPa}$.

The relationship between the sample thickness, pressure at the contact surface at the center, and rotation angle is presented in Fig. 8. Initially, slight changes in the rotation angle cause a large drop of thickness but slight growth of pressure. For example, with an increase of rotation angle from 0 to 0.2 , the thickness reduces by $13 \mu \mathrm{m}$, but pressure grows by only $15 \mathrm{GPa}$. At the later stage of rotation, the increase of rotation angle does not cause a large change of thickness, but causes a very large increase of pressure. It is partially related to the pressure gradient: for large thickness, the pressure gradient and, consequently, pressure are relatively small; however, for small $h, 1 / h$ increases significantly with a slight reduction in $h$, which causes a large increase in pressure gradient and, consequently, pressure. 


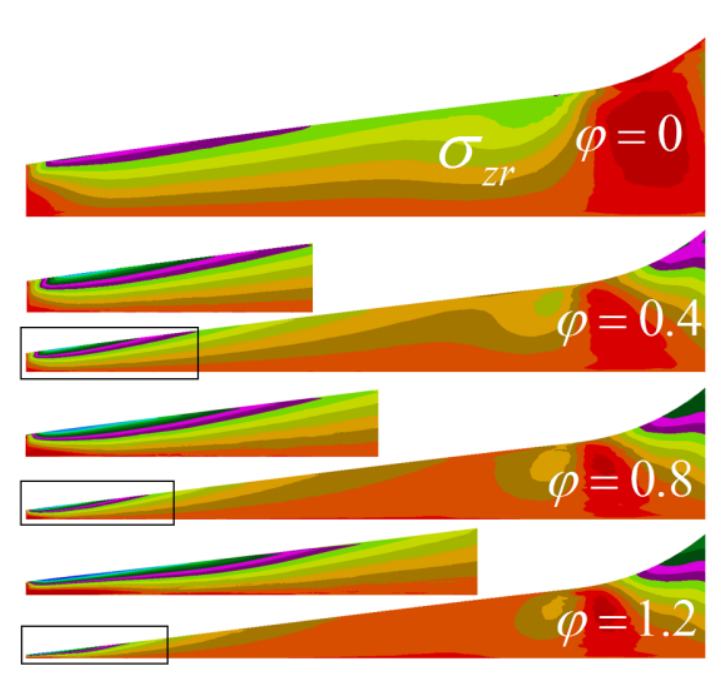

(a)

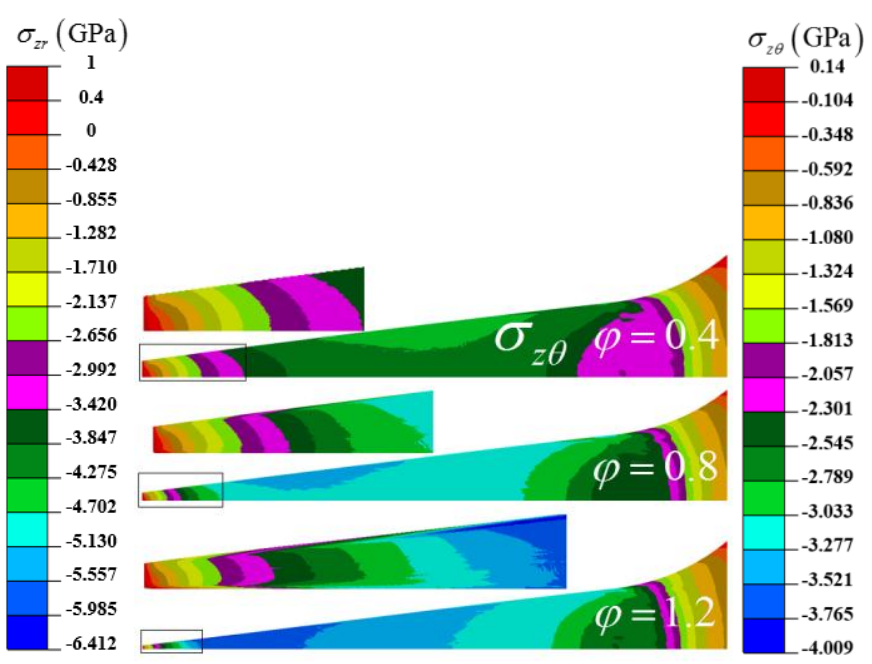

(b)

FIG. 9. Distributions of shear stresses $\sigma_{z r}$ (a) and $\sigma_{z \theta}$ (b) in the sample $(0 \leq r \leq 160 \mu \mathrm{m})$ under a fixed applied normal stress $\sigma_{n}=1.1 \mathrm{GPa}$ with the increasing rotation angle $\varphi$.

The evolution of shear stresses $\sigma_{z r}$ and $\sigma_{z \theta}$ is shown in Fig. 9. Unlike very similar distributions among the different normal stresses, distributions of the shear stresses have completely different patterns. At the symmetry plane, the shear stress $\sigma_{z r}$ is zero due to the symmetry, and it grows to its maximum value at the contact surface. For the anvil slope of $8.5^{\circ}$, the shear stress $\sigma_{z r}$ is very close to friction stress $\tau_{1}$ along the surface, while the $\sigma_{z \theta}$ is very close to the circumferential friction stress $\tau_{2}$. At the periphery, since the pressure $p\left(p \approx \sigma_{c}\right)$ does not change significantly (Fig. 3 (b)), the magnitude of the friction stress $|\tau|=\mu \sigma_{c}=\sqrt{\tau_{1}^{2}+\tau_{2}^{2}}$ essentially does not change as well. During the rotation, $\tau_{2}\left(\sigma_{z \theta}\right)$ grows which causes the reduction of $\tau_{1}\left(\sigma_{z r}\right)$ in order to keep a constant $\mu \sigma_{c}$ at the periphery. In the central region with an increasing rotation angle, the magnitude of the friction stress $|\tau|=\tau_{y}$ increases due to the growth of pressure and consequently the yield strength, which causes the increases of both $\sigma_{z r}$ and $\sigma_{z \theta}$. 


\section{Evolution of stress-strain fields in the diamond}

The stress-strain state of diamond is important from two points of view. First, high stresses cause fracture of diamond and limit the maximum possible pressure. Second, bending of diamonds causes cupping and arrests radial plastic flow in DAC, which does not allow further increase in pressure. While stresses in anvils in DAC were found in Feng, et al., 2016 and references, stresses for RDAC are presented here for the first time. Thus, in this section, we will discuss the stress and strain fields in the diamond anvil. Since stresses are small (below several GPa) due to specific geometry for the major part of an anvil, results will only be shown for the region near the surface contacting the sample, where large stresses and strains appear.

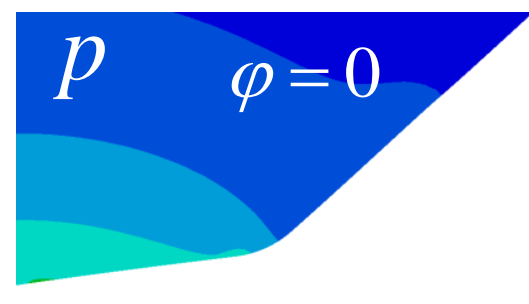

(a)

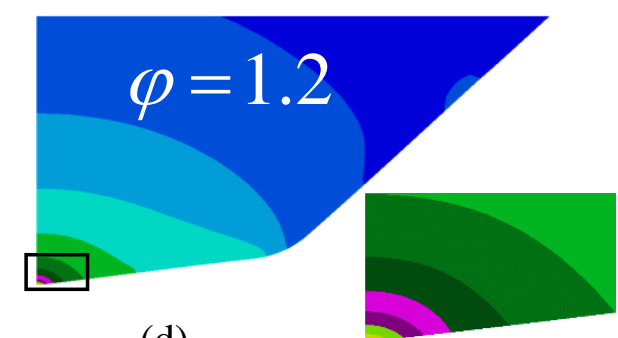

(d)

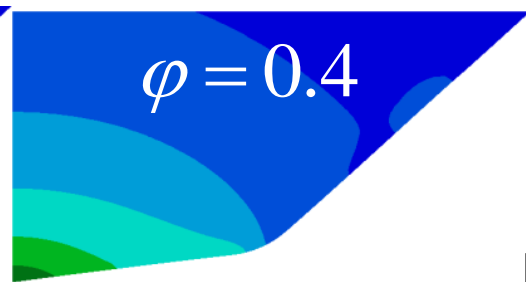

(b)

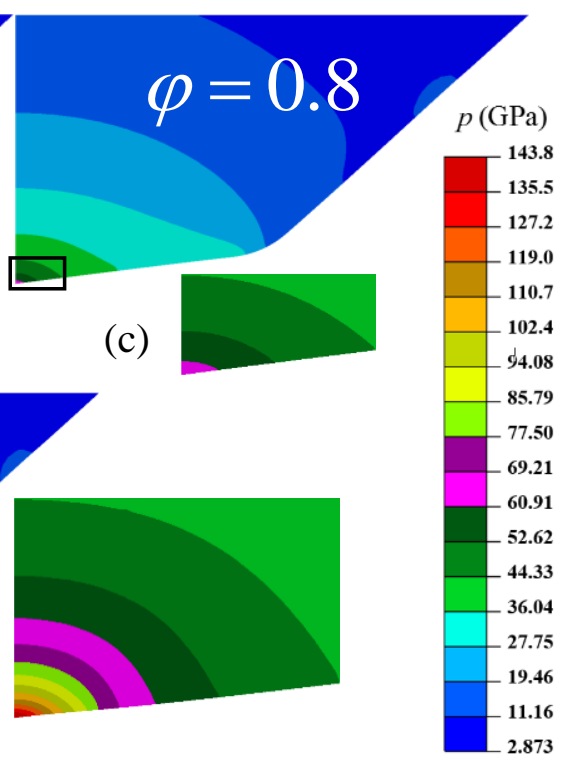

(e)

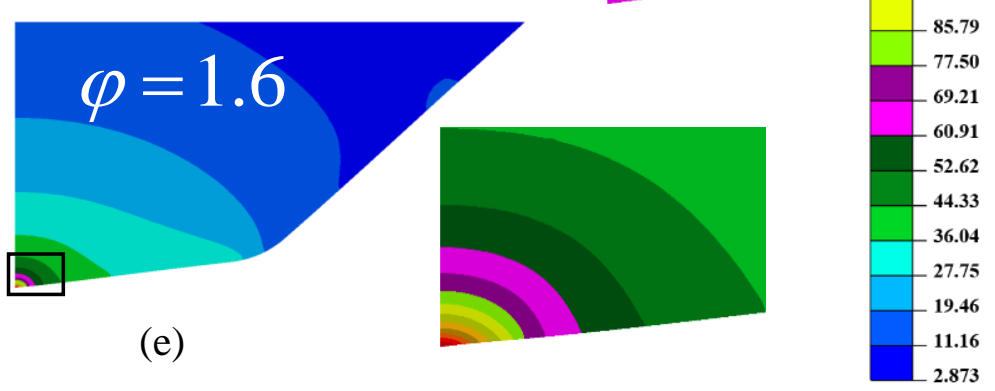

applied normal stress $\sigma_{n}=1.1 \mathrm{GPa}$ with the growing rotation angle $\varphi$. The zoomed central part of a sample is shown near the anvil. 


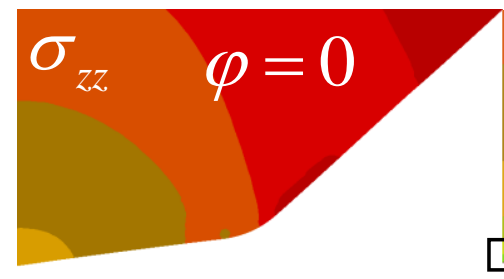

(a)

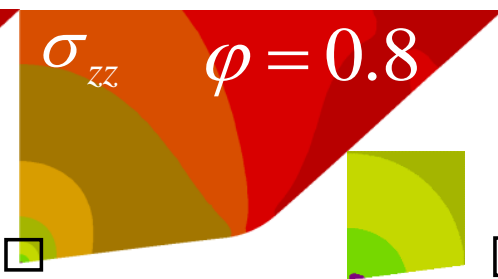

(b)

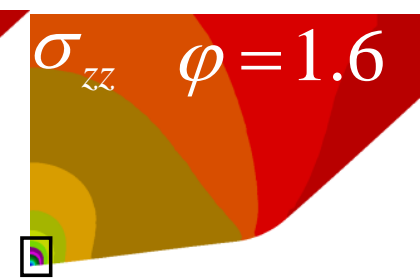

(c)

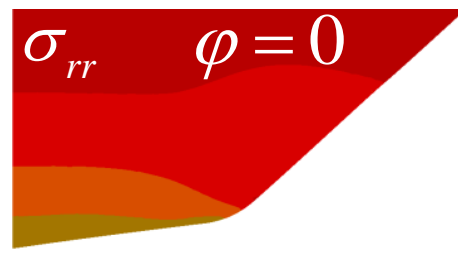

(d)

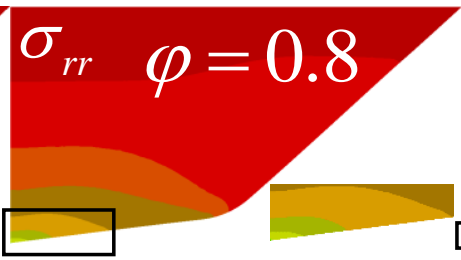

(e)

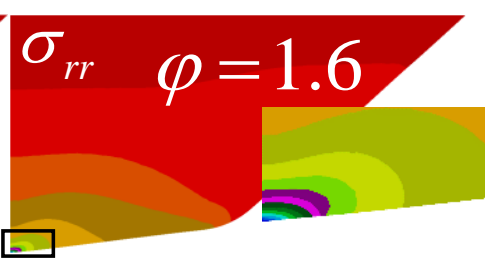

(f)

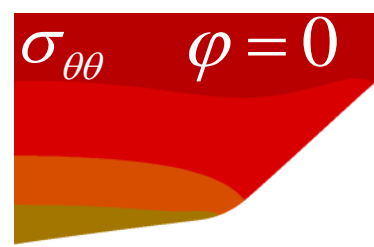

$(\mathrm{g})$

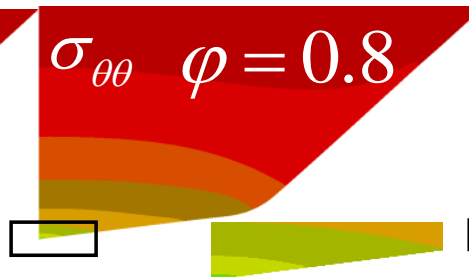

(h)

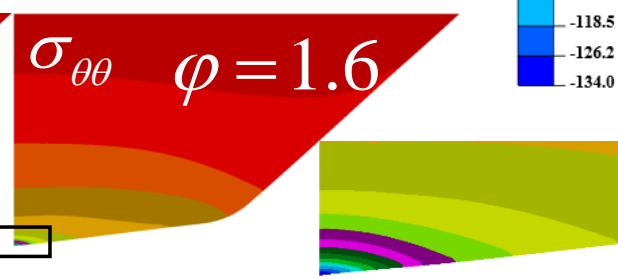

(i)

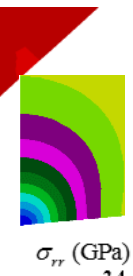

$\sigma_{r r}(\mathrm{GPa})$

$\left[\begin{array}{c}-2.4 \\ --10.14 \\ -17.88\end{array}\right.$

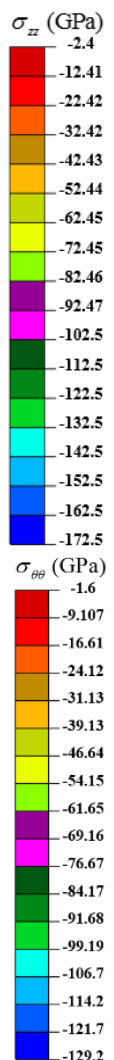

Fig. 11. Distributions of normal stresses $\sigma_{z z}$ (a, b and c), $\sigma_{r r}\left(\mathrm{~d}\right.$, e and f) and $\sigma_{\theta \theta}(\mathrm{g}, \mathrm{h}$, and i) under a fixed applied normal stress $\sigma_{n}=1.1 \mathrm{GPa}$ with the increasing rotation angle $\varphi$.

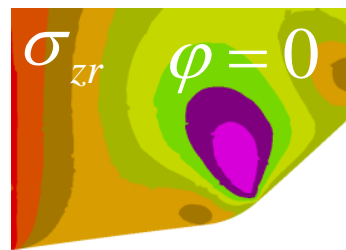

(a)

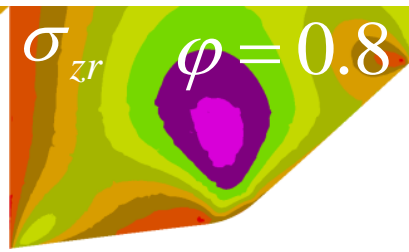

(b)

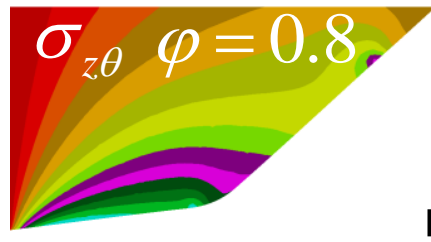

(d)

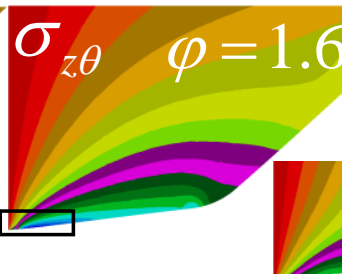

(e)

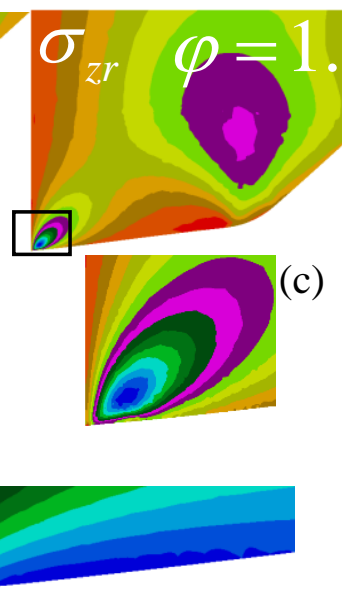

(c)

FIG. 12. Distributions of shear stresses $\sigma_{z r}\left(\mathrm{a}, \mathrm{b}\right.$ and c) and $\sigma_{z \theta}$ (d and e) under a fixed applied normal stress $\sigma_{n}=1.1 \mathrm{GPa}$ with the growing rotation angle $\varphi$. 
Fig. 10 shows the evolution of pressure $p$ in the anvil during the growth of rotation angle $\varphi$. When there is no rotation and under $\sigma_{n}=1.1 \mathrm{GPa}$, pressure is not high and reduces with an increasing coordinate $z$ due to the increase in cross-sectional area, but it does not change significantly along the $r$ axis. When $\varphi$ increases from 0 to 0.4 , pressure grows close to the tip of anvil, which causes the reduction of pressure at the periphery, since the applied normal stress $\sigma_{n}=1.1 \mathrm{GPa}$ is fixed during rotation. With $\varphi$ increasing from 0.4 to 1.6 , the pressure in most of the region does not change. Close to the tip of anvil, pressure grows very intensely and exceeds megabar level at $\varphi=$ 1.6. There is a very large pressure gradient along both the $z$ axis and $r$ axis. Since pressure increases significantly in the small region only, which slightly contributes to the axial force, pressure does not change significantly in most of the region of the anvil. Fields of three normal stresses $\sigma_{z z}, \sigma_{r r}$ and $\sigma_{\theta \theta}$ in the anvil are shown in the Fig. 11. Distinct from the normal stresses in a sample with the very similar distribution patterns, stress $\sigma_{z z}$ changes significantly along both the $z$ and $r$ axes; however, $\sigma_{r r}$ and $\sigma_{\theta \theta}$ are almost homogeneous in the $r$ direction. All normal stresses reduce significantly with an increasing $z$ due to the growth of the cross-sectional area. During the increase of rotation angle from 0.8 to 1.6 , all normal stresses grow significantly only in the region close to the tip of an anvil, which practically does not affect the distributions in other regions. Because a major part of the anvil is under low stresses, the shape of anvil does not change, unlike for the case with DAC in Feng et al, 2016. 


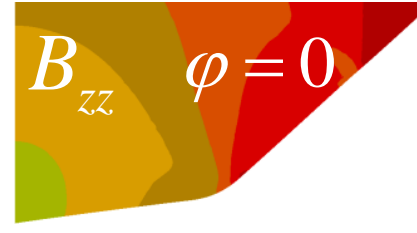

(a)

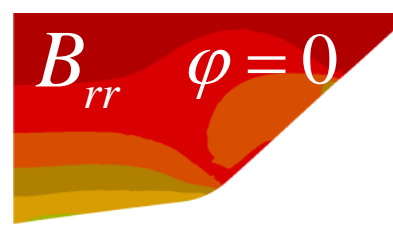

(d)

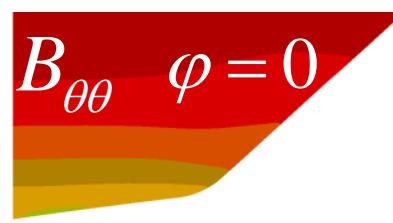

(g)

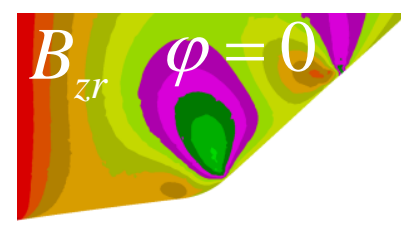

(j)

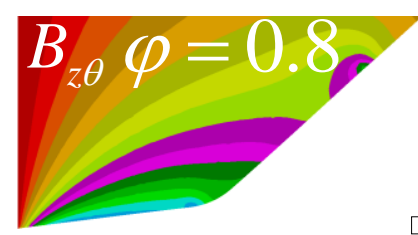

(m)

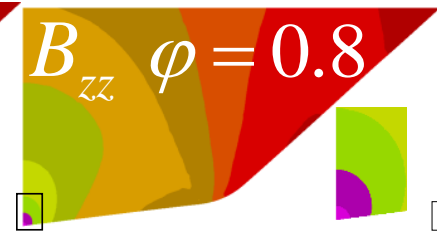

(b)

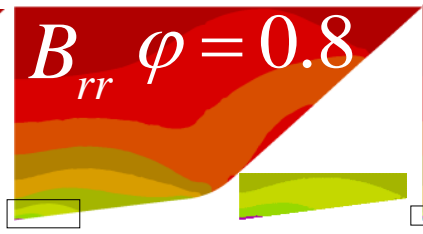

(e)

(f)

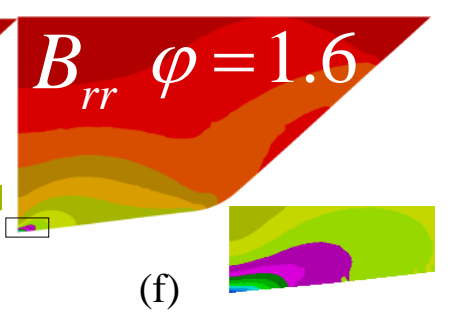

(c)

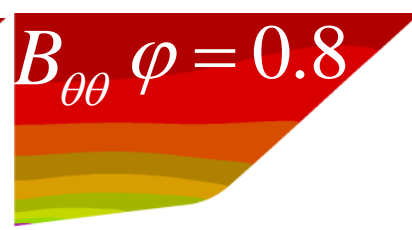

(h)

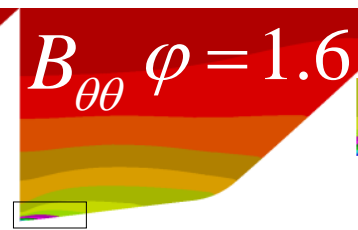

(i)

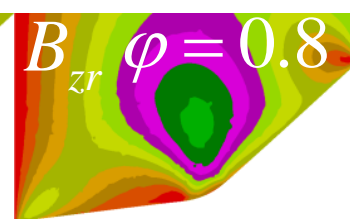

(k)

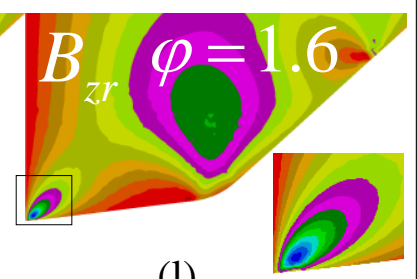

(1)
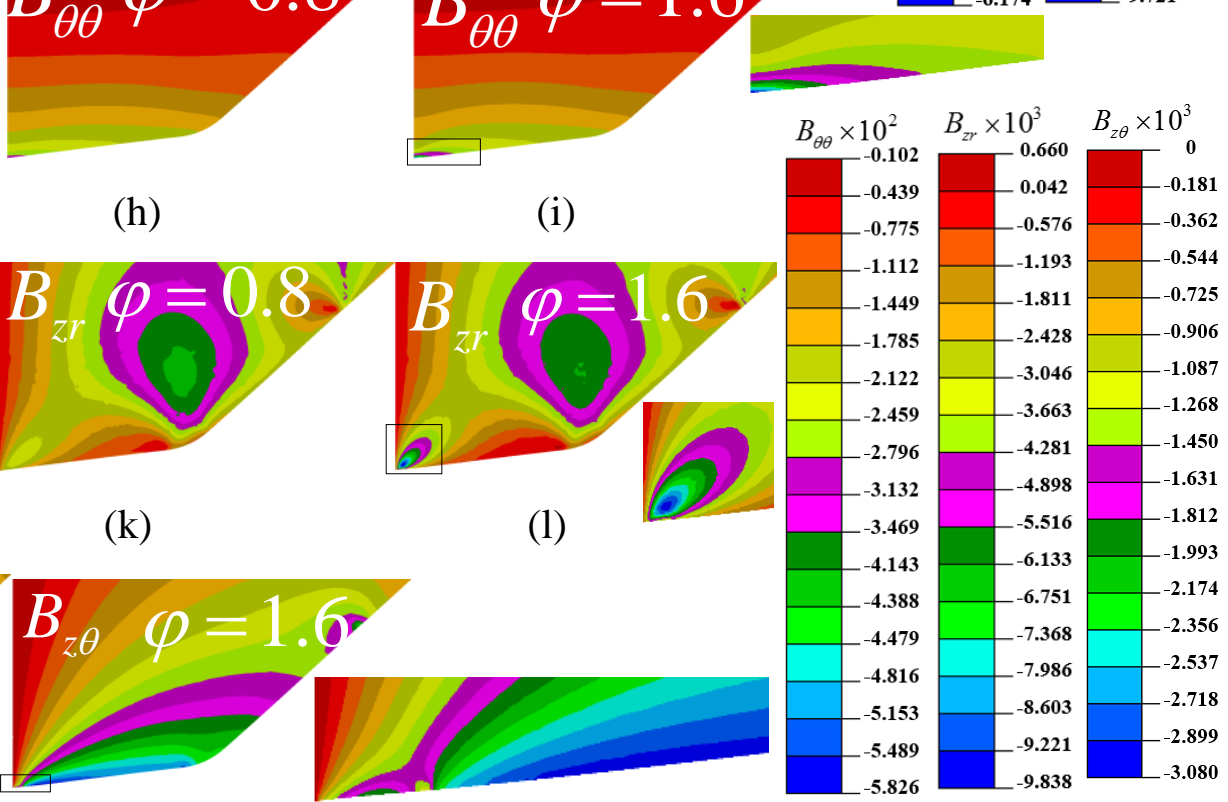

(n)

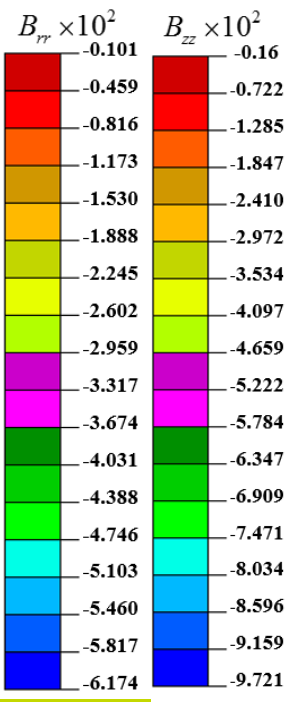

FIG. 13. Distributions of the strains $B_{z z}, B_{r r}, B_{\theta \theta}, B_{z r}$, and $B_{z \theta}$ in the diamond anvil under a fixed applied normal stress $\sigma_{n}=1.1 \mathrm{GPa}$ with the growing rotation angle $\varphi$.

The evolution of shear stresses $\sigma_{z r}$ and $\sigma_{z \theta}$ is shown in Fig. 12 with the growth of rotation angle. The shear stress $\sigma_{z r}$ increases near the tip, but decreases at the periphery. Close to the tip, the shear stress reaches $15.80 \mathrm{GPa}$ slightly away from the contact surface because friction cannot 
surpass the yield strength in shear of a sample at the contact surface. At the periphery, the radial friction stress $\tau_{1}$, or $\sigma_{z r}$, reduces due to the increase of $\tau_{2}$, or $\sigma_{z \theta}$. Initially, $\sigma_{z \theta}$ is zero without rotation, and it continuously grows during rotation. Both shear stresses are highly heterogeneous in both the $r$ and $z$ directions, and the maximum shear stresses are very close to the tip, which is different from the locations of the maximum normal stresses. The evolution of the normal strains $B_{z z}, B_{r r}, B_{\theta \theta}$ and the shear strains $B_{z r}$ and $B_{z \theta}$ is shown in Fig. 13. Distributions have very similar patterns to the corresponding stresses. The maximum $B_{z z}$ in the anvil is around 0.1 , and the use of nonlinear elasticity is important. It follows from Lang et al, 2011 that contributions of the third order terms become visible at pressures above $40 \mathrm{GPa}$. However, the high strain fields are localized near the tip of the anvil only; in the rest of an anvil the strains are relatively small, and the change in shape of an anvil is not visible.

\section{Effects of the loading condition, material parameter, friction coefficient, and the shape of contact surface}

In this section, we will perform the parametric study on the pressure distribution in the sample compressed and twisted in RDAC under different conditions. For interpretation of the results, we will use the simplified equilibrium equation (13), $\frac{d p}{d r}=\frac{2 \tau_{1}}{h}$.

Effect of applied axial force. With an increase in the applied normal stress, $\sigma_{n}$, from $1.0 \mathrm{GPa}$ to $1.18 \mathrm{GPa}$, the pressure expectedly increases in the entire sample for the same rotation angle $(0.8$ or 1.2), see Fig. 14. Major growth in pressure gradient and, consequently, pressure is observed near the center of a sample. This is caused by significant increase in the inverse sample width, $1 / h$, 
at the center due to the anvil's inclined surface for a larger $\sigma_{n}$, by a factor of almost 2 . In addition, with a larger $p$ for $\sigma_{n}=1.18 \mathrm{GPa}$, the yield strength and friction stress at the center increase, which in return causes the increases of pressure gradient and pressure. However, this effect is weaker than the effect of increase in $1 / h$ (Fig. 14). Even when the pressure reaches 240GPa at the center, it is still relatively small at the periphery. Due to this, the angle between the contact surface and the radial axis $r$ is 6.6 degrees at $r=120 \mu \mathrm{m}$ in RDAC, which is much larger than 3.1 degrees in DAC. Therefore, the cupping phenomenon can be reduced significantly in RDAC, which leads to the possibility of reaching much higher pressure.

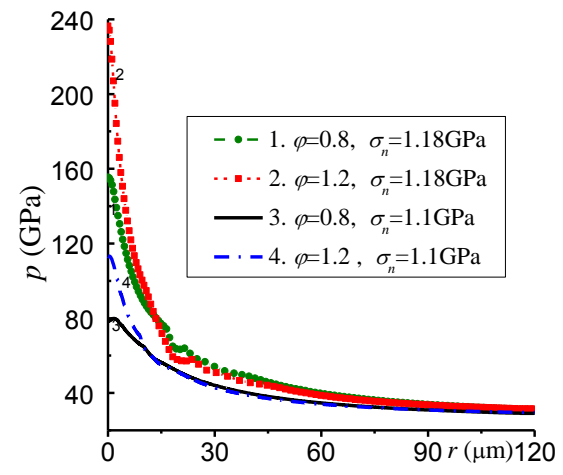

(a)

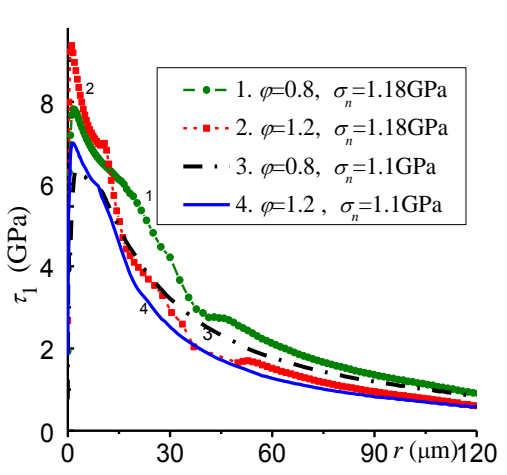

(b)

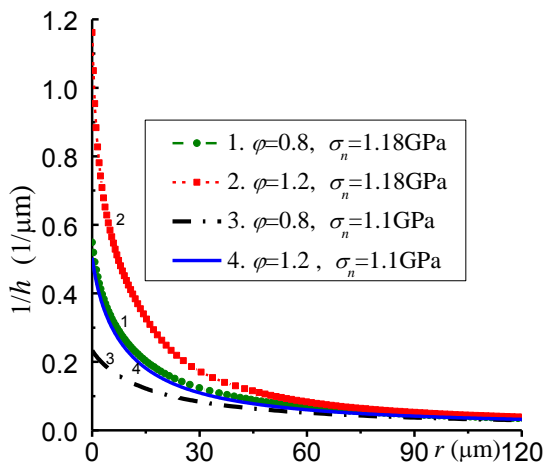

(c)

Fig. 14. Distributions of pressure $p$ (a) and friction stress $\tau_{1}$ (b) at the contact surface, and inverse thickness $1 / h$ of sample (c) under fixed applied normal stress $\sigma_{n}=1.1 \mathrm{GPa}$ (curves (3) and (4)) and $\sigma_{n}=1.18 \mathrm{GPa}$ (curve (1) and (2)), for two rotation angles $\varphi$.

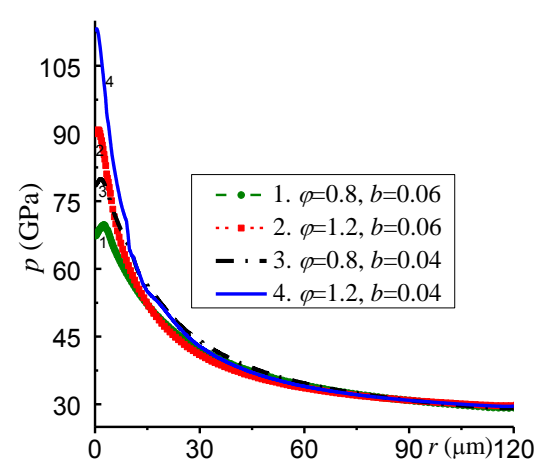

(a)

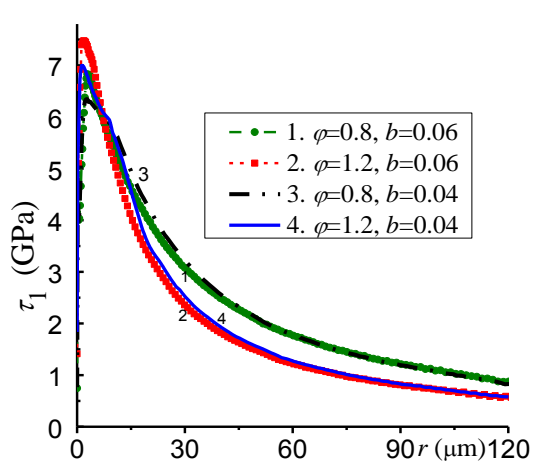

(b)

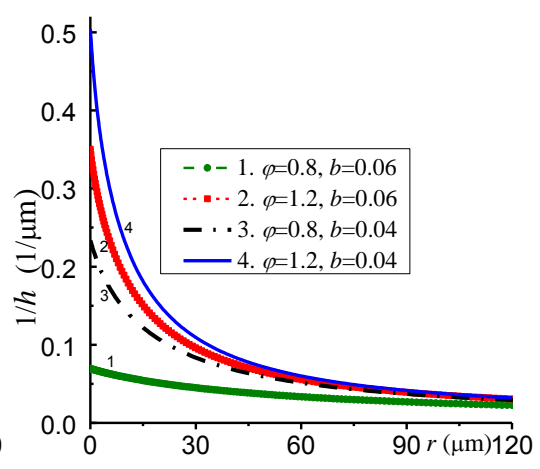

(c)

FIG. 15. Distributions of pressure $p$ (a) and friction stress $\tau_{1}$ (b) at the contact surface, and inverse thickness $1 / h$ of sample (c) under fixed applied normal stress $\sigma_{n}=1.1 \mathrm{GPa}$, for different 
pressure dependence coefficients $b=0.04$ (curves (3) and (4)) and $b=0.06$ (curves (1) and (2)), for two rotation angles $\varphi$.

Effect of the pressure dependence of the yield strength $\sigma_{y}(p)=\sigma_{y 0}+b p$. Intuitively, an increase in $b$ should increase friction stress at the center (which is indeed true, see Fig. 15b) and, consequently, pressure gradient and pressure at the center (which, however, is not the case). The reason is that the increase in the yield strength slows down the material radial flow and reduction in thickness (Fig. 15 (c)). For $b=0.04$ the inverse thickness $1 / h$ is much larger than that for $b=$ 0.06, which makes a much stronger and opposite contribution to the pressure gradient at the center of a sample than the increase in friction stress. Consequently, in Fig. 15 (a), pressure at the center is much larger for a smaller $b$ due to a larger $1 / h$ (Fig. 15). Similarly, when we reduced $\sigma_{y 0}$ from $8 \mathrm{GPa}$ to $7 \mathrm{GPa}$, we obtained larger pressure at the center due to the same reason as for reduction in $b$. Thus, while counterintuitive, this effect can be properly rationalized.

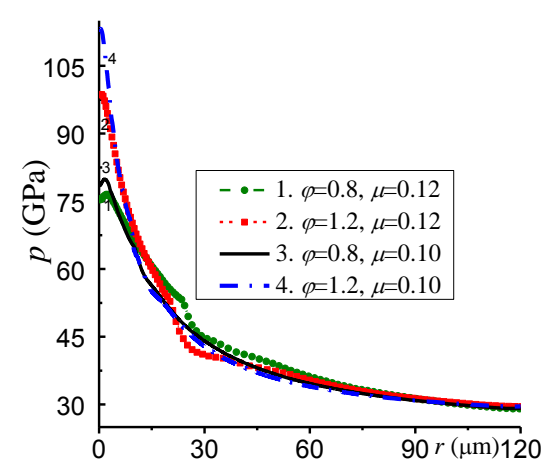

(b)

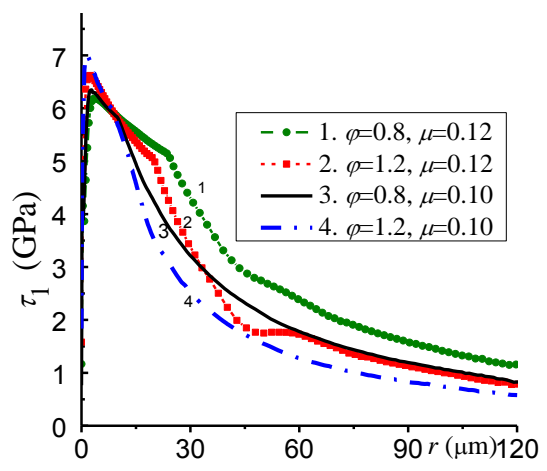

(b)

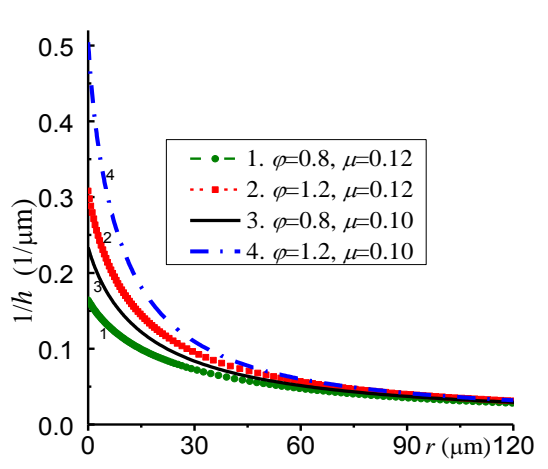

(c)

Fig. 16. Distributions of the pressure $p$ (a) and friction stress $\tau_{1}$ (b) at the contact surface, and inverse thickness $1 / h$ of sample (c) under a fixed applied normal stress $\sigma_{n}=1.1 \mathrm{GPa}$, with the friction coefficient $\mu=0.10$ (curves (3) and (4)) and $\mu=0.12$ (curves (1) and (2)) for two different rotation angles $\varphi$.

Effect of friction coefficient. The situation is similar to that for the effect of the yield strength. Intuitively, an increase in friction coefficient should increase friction stress (which is true except for a very small central region, see Fig. 16 (b)) and its contribution to the pressure gradient and 
maximum pressure. However, because axial force is fixed, an increase in friction increases sample thickness and strongly reduces $1 / h$ (Fig. 16 (c)). When $\mu$ increases from 0.1 to 0.12 , the resultant pressure gradient and pressure slightly change in the major part of a sample, but reduce at the center, especially for $\varphi=1.2$.

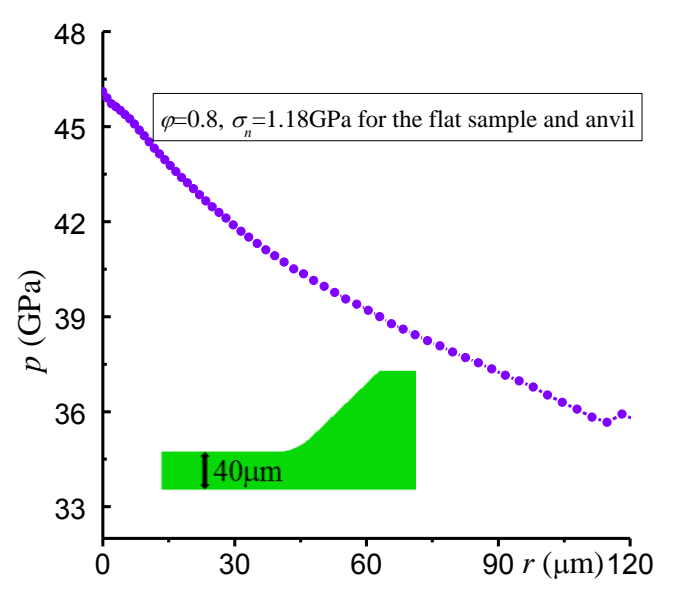

Fig. 17. Distributions of the pressure $p$ at the contact surface at rotation angle $\varphi=0.8$ and $\sigma_{n}=1.18 \mathrm{GPa}$ for a flat anvil and a sample with an initial thickness $h=80 \mu \mathrm{m}$.

Flat anvil. The pressure distribution for the flat sample and anvil in Fig. 17 should be compared with the pressure for inclined surface for the same $\sigma_{n}=1.18 \mathrm{GPa}$ and $\varphi=0.8$ (green curve 1 in Fig. 14 (a)). Obviously, pressure is much more homogenous, and maximum pressure is more than 3 times lower with a flat anvil. One of the reasons is that with an inclined anvil, sample thickness is decreasing at the same axial force due to smaller resistance to the radial plastic flow, which causes a larger pressure gradient. However, the main reason is the large gradient in $1 / h$ in Fig. 14 (c) with the maximum of $1 / h$ at the center of the sample, which leads to a larger pressure gradient and larger pressure at the center. For a flat anvil, the thickness gradient is almost zero since deformation of the anvil is small due to a low pressure, which causes a more homogenous pressure distribution. Pressure self-focusing effect is not possible for flat anvils. 
Note that there are significant efforts in studying materials' dynamic behavior under high pressure and large shear deformations. A review of experimental techniques under high rate deformation can be found in (Field et al. 2004), which covers dropweight machines, split Hopkinson pressure bars, Taylor impact and shock loading by plate impact. Metals subjected to the weak shock waves experience strain rates in the range of $10^{4}-10^{8} \mathrm{~s}^{-1}$, and for overdriven shocks the strain rate range is $10^{9}-10^{12} \mathrm{~s}^{-1}$ (Austin and McDowell, 2011). Pressure-shear plate impact (PSPI) experiments have been performed by Clifton and co-workers to measure the flow stress of $\mathrm{Cu}$ (Frutschy and Clifton, 1998; Tong et al., 1995; Huang and Clifton, 1985) and $\mathrm{Al}(\mathrm{Li}, 1981)$ at the strain rates in the range of $10^{5}-10^{6} \mathrm{~s}^{-1}$. Recently, Jiao and Clifton (2017) conducted the PSPI experiments to study the mechanical response of an elastomer (polyurea) at pressures up to $18 \mathrm{GPa}$. Due to high strain rate, in-situ experiments under shock wave loadings are much more challenging than those in DAC, and the surface velocity is most often measured in the flyer-target impact experiments. The DAC allows one to perform an in-situ study by using advanced diagnostics such as optical, Raman, and $\mathrm{x}$-ray techniques. In addition, the information of the distribution of a sample thickness, pressure, and concentration of a high pressure phase (Hemley et al., 1997, Levitas et al., 2006) can be obtained. Pressure can be measured up to 750 GPa (Dubrovinsky et al., 2015). Since viscoplastic constitutive equations should transform for small strain rate to the rate-independent plasticity, studies at static pressures and large plastic shear are important initial steps in developing constitutive equations for dynamic loading.

\section{Concluding remarks}

In this paper, the first modeling results for plastic flow and evolution of the stress-strain state for a rhenium sample compressed and twisted in RDAC under megabar pressures are 
presented. This problem is crucial for the development of high pressure science and technologies. From a mechanical point of view, this is a quite complex three-dimensional problem with multiple nonlinearities. Thus, sample thickness is reduced by a factor of 90 , pressure reaches $240 \mathrm{GPa}$, and even elastic strain in diamond reaches 0.1 , all of which require nonlinear elasticity for cubic crystals. We utilize the thermodynamically consistent equations for large elastic and plastic strains developed in Levitas, 1996 and Feng et al., 2016 and the FEM algorithms presented in Feng et al., 2016. The pressure dependence of elastic and plastic properties were taken into account. Contact sliding between sample and anvil in a two-dimensional setting obeying Colulomb friction was implemented. That means the current work represents, in particular, verification of the effectiveness of the model (and especially the numerical algorithm developed in Feng et al., 2016) for more general and complex cases of: three-dimensional loading, two-dimensional contact sliding, much larger plastic strains and pressure (and stress), and plastic strain gradients. All material parameters (elastic and plastic constants and friction coefficient) have been calibrated in Feng et al., 2016 for the best reproduction of experimental results in Hemley et al., 1997. Obtained results represent not only important quantitative information about evolution of the stress-strain fields in a sample and an anvil, but also nontrivial qualitative results. Thus, a huge pressure gradient leading to high pressure was found near the center of a sample. This phenomenon was called the pressure self-focusing effect. The main conditions for existence of the pressure selffocusing effect are: beveled anvils and small thickness of a sample at the center leading to large gradient of the sample thickness. For flat anvils or relatively large sample thickness, pressure selffocusing cannot occur when the thickness gradient is absent or small. This may explain why this effect was not yet observed in previous experiments. However, increased pressure gradient at the center was observed after compression in DAC in Hemley et al., 1997 when thickness reached 6 
microns. Feng et al., 2016 used the same model as here and reproduced pressure distribution from Hemley et al., 1997. This can be considered a conceptual experimental confirmation of the pressure self-focusing. Intuitively, an increase in the yield strength, its pressure dependence, and friction coefficient should increase pressure gradient. However, for the same applied force, these lead to a larger sample thickness and thickness gradient, which in total reduces pressure gradient at the center.

For the same axial force, the thickness of the sample after rotation in RDAC is much smaller than for compression in DAC. That it is why sample thickness gradient and, consequently, pressure gradient is much larger in RDAC than in DAC; maximum pressure is significantly larger as well. Also, since high pressure is localized in a very narrow region both in a sample and an anvil, there is a much smaller probability to meet a critical defect in this region, which will cause fracture of an anvil. Due to the strong localization of high stresses, deformation and bending of an anvil are much smaller for RDAC than for DAC. The main factor limiting increase in maximum pressure in DAC is bending of an anvil, which causes cupping of the anvil and sample and arrests radial plastic flow. In contrast, cupping was not observed (at least up to $240 \mathrm{GPa}$ ), and reduction of a beveling angle was from $8.5^{\circ}$ to $6.5^{\circ}$ in RDAC. Consequently, rotation of an anvil is not only a way to superimpose large plastic shear on high pressure to promote PTs and chemical reactions, but it is also an effective way to increase a maximum achievable pressure, although, in a small volume.

Also, we found that one of the traditional methods of determining the pressure dependence of the yield strength based on measured force and torque (Bridgman 1935 and 1952; Vereschagin et al., 1971) possesses significant error. Torque for a perfectly plastic material grows up to maximum rotation of 1.6 radians that we applied due to redistribution between radial and 
circumferential friction stresses. Thus, saturation (reaching maximum value and remaining unchanged) of torque cannot be interpreted as saturation of strain hardening. Also, due to Coulomb friction, the magnitude of the shear friction stress is smaller than the yield strength in shear under a given pressure.

The developed model and obtained results represent a new computational tool for the optimum design of geometry and loading conditions in experiments under extreme pressure and large plastic deformations. They also develop new intuition in mechanical structural responses at megabar pressures. Simplified versions of this model and the same numerical algorithms can be used to simulate HPT at lower pressure. As the next step, we plan to include strain-induced PTs to be able to perform simulations similar to those in Feng and Levitas, 2013; Feng and Levitas, 2016; Feng et al., 2014; Levitas and Zarechnyy, 2010a but at much higher pressure.

\section{Acknowledgements}

The support of NSF (DMR-1434613), ARO (W911NF-12-1-0340), and Iowa State University (Schafer 2050 Challenge Professorship) is gratefully acknowledged.

\section{References}

1. Alexandrova, M. M., Blank, V. D., and Buga, S. G., 1993. Phase-transitions in Ge and Si under shear-stress at pressures up to 12 Gpa and P-T-gamma diagrams of these elements. Solid State Phys. 35, 1308-1317.

2. Austin R. A., and McDowell D. L., 2011. A dislocation-based constitutive model for viscoplastic deformation of fcc metals at very high strain rates. Int. J. Plast. 27, 1-24.

3. Beygelzimer, Y., · Kulagin, R., · Toth, L. S., and- Ivanisenko, Y. 2016. The self-similarity theory of high pressure torsion. Beilstein J. Nanotechnol. 7, 1267-1277.

4. Blank, V. D., Boguslavsky, Y. Y., Eremets, M. I., Itskevich, E. S., Konyaev, Y. S., Shirokov, A. M., and Estrin, E. I., 1984. Pressure self-multiplication effect on phase-transition under quasi-hydrostatic conditions. Zh. Eksp. Teor. Fiz. 87, 922-926.

5. Blank, V. D., and Estrin, E. I., 2014. Phase transitions in solids under high Pressure, CRC Press, New York. 
6. Bridgman, P. W., 1935. Effect of high shear stress combined with high hydrostatic pressure, Phys. Rev. 48, $825-847$.

7. Bridgman P. W., 1937. Shearing phenomena at high pressures, Particularly in inorganic compounds, Proc. Am. Acad. Arts Sci. 71, 387-460.

8. Bridgman P. W., 1947. The effect of high mechanical stress on certain solid explosives, J. Chem. Phys. 15, 311-313.

9. Bridgman, P. W., 1952. Studies in large plastic flow and fracture with special emphasis on the effects of hydrostatic pressure. McGraw-Hill, New York.

10. Chen, B., Lutker, K. Raju, S. V., Yan, J., et al., 2012. Pressure-promoted texturing in nanocrystalline nickle: Probing the lower size limit of dislocation activity. Science 338, 1448-1451.

11. Borodachenkova, M., Barlat, F., Wen, W., Bastos, A., Gracio, J. J., 2015. A microstructure-based model for describing the material properties of Al-Zn alloys during high pressure torsion. Int. J. Plast. 68, 150-163.

12. Drozdov A. P., Eremets M. I., Troyan I. A., Ksenofontov V., and Shylin S. I., 2015. Conventional superconductivity at 203 kelvin at high pressures in the sulfur hydride system. Nature 525, 73-76.

13. Dubrovinsky, L., Dubrovinskaia, N., Prakapenka, V. B., and Abakumov, A. M., 2012. Implementation of micro-ball nanodiamond anvils for high-pressure studies above 6 Mbar. Nat. Commun. 3, 1163.

14. Dubrovinsky, L., Dubrovinskaia, N., Bykova, E., Bykov, M., Prakapenka, V., Prescher, C., Glazyrin, K., Liermann, H. P., Hanfland, M., Ekholm, M., Feng, Q., Pourovskii, L. V., Katsnelson, M. I., Wills, J. M., and Abrikosov, I. A., 2015. The most incompressible metal osmium at static pressures above 750 gigapascals. Nature 525, 226-229.

15. Edalati, K., Horita, Z., 2016. A review on high-pressure torsion (HPT) from 1935 to 1988. Mat. Sci. Eng. A. $652,325-352$.

16. Edalati K., Lee D. J., Nagaoka T., Arita M., Kim H. S., Horita Z., and Pippan R. 2016. Real hydrostatic pressure in high-pressure torsion measured by bismuth phase transformations and FEM simulations, Mater. Trans. 57, 533-538.

17. Enikolopyan, N. S., 1985. Some aspects of chemistry and physics of plastic flow. Pure Appl. Chem. $57,1707$.

18. Enikolopyan, N. S., 1989. Super-fast chemical reactions in solids. Russ. J. Phys. Chem. 63, 1261.

19. Eremets, M. I., 1996. High pressure experimental methods. Oxford University, Oxford; New York.

20. Feng, B., and Levitas, V. I., 2013. Coupled phase transformations and plastic flows under torsion at high pressure in rotational diamond anvil cell: Effect of contact sliding. J. Appl. Phys. 114, 213514.

21. Feng, B., and Levitas, V. I., 2016. Effects of gasket on coupled plastic flow and strain-induced phase transformations under high pressure and large torsion in a rotational diamond anvil cell. J. Appl. Phys. 119, 015902.

22. Feng, B., and Levitas, V. I., 2017. Plastic flows and strain-induced alpha to omega phase transformation in zirconium during compression in a diamond anvil cell: Finite element simulations. Mat. Sci. Eng. A. 680, 130140.

23. Feng, B., Levitas, V. I., and Hemley, R. J., 2016. Large elastoplasticity under static megabar pressures: formulation and application to compression of samples in diamond anvil cells. Int. J. Plast. 84, 33-57. 
24. Feng, B., Levitas, V. I., and Zarechnyy, O. M., 2014. Strain-induced phase transformations under high pressure and large shear in a rotational diamond anvil cell: simulation of loading, unloading, and reloading. Comp. Mater. Sci. 84, 404-416.

25. Field, J. E., Walley, S. M., Proud, W. G., Goldrein, H. T., and C.R. Siviour, 2004. Review of experimental techniques for high rate deformation and shock studies. Int. J. Impact. Eng. 30, 725-775.

26. Figueiredo, R. B., Cetlin, P. R., and Langdon, T. G., 2011. Using finite element modeling to examine the flow processes in quasi-constrained high-pressure torsion. Mat. Sci. Eng. A. 528, 8198-8204.

27. Frutschy, K. J., and Clifton, R. J., 1998. High-temperature pressure-shear plate impact experiments on OFHC copper. J. Mech. Phys. Solids 46, 1723-1743.

28. Hemley, R. J., Mao, H. K., Shen, G. Y., Badro, J., Gillet, P., Hanfland, M., and Hausermann, D., 1997. X-ray imaging of stress and strain of diamond, iron, and tungsten at megabar pressures. Science 276, 1242-1245.

29. Hammond, C. R., 2004. "The Elements". In Handbook of Chemistry and Physics (81st ed.). CRC press, Boca Raton.

30. Huang, S., and Clifton, R. J., 1985. In: Kawata, K., Shioiri, J. (Eds.), Macro- and Micro-Mechanics of High Velocity Deformation and Fracture. IUTAM, Tokyo, p. 63.

31. Jeanloz, R., Godwal, B. K., and Meade, C., 1991. Static Strength and Equation of State of rhenium at ultrahigh pressures. Nature 349, 687-689.

32. Ji, C., Levitas, V. I., Zhu, H. Y., Chaudhuri, J., Marathe, A., and Ma, Y.Z., 2012. Shear-induced phase transition of nanocrystalline hexagonal boron nitride to wurtzitic structure at room temperature and lower pressure. P. Natl. Acad. Sci. USA 109, 19108-19112.

33. Jiao, T. and Clifton, R. J., 2017. Experimental and computational investigation of the response of an elastomer at pressures up to $18 \mathrm{GPa}$ and strain rates of $105-106 \mathrm{~s}^{-1}$. AIP Conference Proceedings 1793, 140008.

34. Kaercher, P., Speziale, S., Miyagi, L., Kanitpanyacharoen, W., and Wenk, H. R., 2012. Crystallographic preferred orientation in wustite $(\mathrm{FeO})$ through the cubic-to-rhombohedral phase transition. Phys. Chem. Minerals 39, 613-626.

35. Kawasaki, M., and Langdon, T. G., 2014. Review: achieving superplasticity in metals processed by highpressure torsion. J. Mater. Sci. 49, 6487-6496.

36. Kim, H. S., 2001. Finite element analysis of high pressure torsion processing. J. Mater. Process. Tech. 113, 617-621.

37. Kim, H. S., Hong, S. I., Lee, Y. S., Dubravina, A. A., and Alexandrov, I. V., 2003. Deformation behavior of copper during a high pressure torsion process. J. Mater. Process. Tech. 142, 334-337.

38. Lang, J. M., and Gupta, Y. M., 2011. Experimental Determination of Third-Order Elastic Constants of Diamond. Phys. Rev. Lett. 106, 125502.

39. Levitas, V. I., 1996. Large Deformation of materials with complex rheological properties at normal and high pressure. Nova Science Publishers, New York.

40. Levitas, V. I., 2004a. High-pressure mechanochemistry: Conceptual multiscale theory and interpretation of experiments. Phys. Rev. B 70, 184118.

41. Levitas, V. I., 2004b. "Continuum mechanical fundamentals of mechanochemistry," in High-pressure surface science and engineering, edited by Y. Gogotsi and V. Domnich, Institute of Physics Pub, Bristol, Philadelphia. 
42. Levitas, V. I., Ma, Y. Z., Hashemi, J., Holtz, M., and Guven, N., 2006. Strain-induced disorder, phase transformations, and transformation-induced plasticity in hexagonal boron nitride under compression and shear in a rotational diamond anvil cell: In situ x-ray diffraction study and modeling. J. Chem. Phys. 125, 044507.

43. Levitas, V. I., Ma, Y. Z., Selvi, E., Wu, J. Z., and Patten, J. A., 2012. High-density amorphous phase of silicon carbide obtained under large plastic shear and high pressure. Phys. Rev. B 85, 054114.

44. Levitas, V. I., and Zarechnyy, O. M., 2006. Kinetics of strain-induced structural changes under high pressure. J. Phys. Chem. B 110, 16035-16046.

45. Levitas, V. I., and Zarechnyy, O. M., 2010a. Modeling and simulation of strain-induced phase transformations under compression and torsion in a rotational diamond anvil cell. Phys. Rev. B 82, 174124.

46. Levitas, V.I., and Zarechnyy, O.M., 2010b. Numerical study of stress and plastic strain evolution under compression and shear of a sample in a rotational anvil cell, High. Press. Res. 30, 653-669.

47. Li, C.H., 1981. Ph.D. thesis, Brown University.

48. Lv, M. B., Cheng, Y., Qi, Y. Y., Ji, G. F., and Piao, C. G., 2012. Elastic properties and phonon dispersions of rhenium in hexagonal-close-packed structure under pressure from first principles. Physica B 407, 778-783.

49. Manghnani, M. H., Katahara, K., and Fisher, E. S., 1974. Ultrasonic equation of state of rhenium. Phys. Rev. B 9, 1421-1431.

50. Merkel, S., Liermann, H. P., Miyagi, L., and Wenk, H. R., 2013. In situ radial x-ray diffraction study of texture and stress during phase transformations in bcc-, fcc- and hcp-iron up to $36 \mathrm{GPa}$ and $1000 \mathrm{~K}$. Acta Mater. 61, $5144-5151$

51. Merkel, S., Kubo, A., Miyagi, L., Speziale, S., Duffy, T. S. Mao, H. K., and Wenk, H. R., 2006. Plastic deformation of $\mathrm{MgGeO} 3$ post-perovskite at lower mantle pressures. Science 311, 644-646.

52. Nielsen, O. H., 1986. Optical phonons and elasticity of diamond at megabar stresses. Phys. Rev. B 34, 58085819.

53. Novikov, N. V., Polotnyak S.B., Shvedov L.K., and Levitas, V.I., 1999, Regularities of Phase transitions under compression and shear in diamond anvils: Experiment and theory. J. of Superhard Mater. 3, 39-51.

54. Perez-Prado, M. T., and Zhilyaev, A. P., 2009. First experimental observation of shear induced hcp to bcc transformation in Pure Zr. Phys. Rev. Lett. 102, 175504.

55. Singh, A. K., Hu, J., Shu, J., Mao, H. K., and Hemley, R. J., 2012. Strength of rhenium from x-ray diffraction experiments under nonhydrostatic compression to $250 \mathrm{GPa}$. J. Phys. Conf. Ser. 377, 012008.

56. Srinivasarao, B., Zhilyaev, A. P., Gutierrez-Urrutia, I., and Perez-Prado, M. T., 2013. Stabilization of metastable phases in Mg-Li alloys by high-pressure torsion. Scripta Mater. 68, 583-586.

57. Thomsen, E. G., Yang, C. T., and Kobayashi, S. O., 1965. Mechanics of Plastic Deformation in Metal Processing. Macmillan, New York.

58. Troyan I., Gavriliuk A., Rueffer R., Chumakov A., Mironovich A., Lyubutin I., Perekalin D., Drozdov A., and Eremets M.I. , 2016. Obervation of superconductivity in hydrogen sulfide from nuclear resonant scattering. Science, 351, 1303-1306.

59. Tong, W., Clifton, R. J., and Huang, S., 1992. Pressure-shear impact investigation of strain rate history effects in oxygen-free high-conductivity copper. J. Mech. Phys. Solids 40, 1251-1294. 
60. Valiev R. Z., Estrin, Y., Horita, Z., Langdon, T.G., Zehetbauer, M.J., and Zhu, Y.T. 2016a. Producing bulk ultrafine-grained materials by severe plastic deformation: ten years later bulk nanostructured materials from severe plastic deformation. JOM 68, 1216-1226.

61. Valiev R. Z., Estrin, Y., Horita, Z., Langdon, T.G., Zehetbauer, M.J., and Zhu, Y.T. 2016b. Fundamentals of Superior Properties in Bulk NanoSPD Materials. Mater. Research Letter 4, 1-21.

62. Vereschagin, L. F., Zubova, E. V., Burdina K. P., and Aparnikov, G. L., 1971. Dokl. Akad. Nauk SSSR 196, $81-82$.

63. Vohra, Y. K., Duclos, S. J., and Ruoff, A. L., 1987. High-pressure x-ray-diffraction studies on rhenium up to 216 GPa (2.16 Mbar). Phys. Rev. B 36, 9790-9792

64. Wang, K., Zhu, W., Xiao, S., Chen, K., Deng, H., and Hu, W., 2015. Coupling between plasticity and phase transition of polycrystalline iron under shock compressions. Int. J. Plast. 71, 218-236.

65. Weir, S. T., Akella, J., Ruddle, C., Goodwin, T., and Hsiung, L., 1998. Static strengths of Ta and U under ultrahigh pressures. Phys. Rev. B 58, 11258-11265.

66. Yoon, S. C., Horita, Z., and Kim, H. S., 2008. Finite element analysis of plastic deformation behavior during high pressure torsion processing. Journal of Materials Processing Technology 201, 32-36.

67. Zharov, A., 1994. High Pressure Chemistry and Physics of Polymers. Kovarskii, Ed., CRC Press, Boca Raton, 267-301.

68. Zhao, Y. and Zhang. J., 2007. Enhancement of yield strength in zirconium metal through high-pressure induced structural phase transition. In-situ observation of texture changes during phase transformations in ultra-lowcarbon steel. Metall. Mater. Appl. Phys. Lett. 91, 201907. 